\title{
Les polémiques sur la perception entre stoïciens et académiciens
}

Jean-Baptiste Gourinat

\section{(2) OpenEdition}

1 Journals

Édition électronique

URL : https://journals.openedition.org/philosant/932

DOI : 10.4000/philosant.932

ISSN : 2648-2789

Éditeur

Éditions Vrin

\section{Édition imprimée}

Date de publication : 1 novembre 2012

Pagination : 43-88

ISBN : 978-2-7574-0400-3

ISSN : 1634-4561

Référence électronique

Jean-Baptiste Gourinat, « Les polémiques sur la perception entre stoïciens et académiciens », Philosophie antique [En ligne], 12 | 2012, mis en ligne le 01 novembre 2018, consulté le 03 décembre 2022. URL : http://journals.openedition.org/philosant/932 ; DOI : https://doi.org/10.4000/philosant. 932

\section{(c) (i) () $\Theta$}

Creative Commons - Attribution - Pas d'Utilisation Commerciale - Pas de Modification 4.0 International - CC BY-NC-ND 4.0

https://creativecommons.org/licenses/by-nc-nd/4.0/ 


\section{LES POLÉMIQUES SUR LA PERCEPTION ENTRE STOÏCIENS ET ACADÉMICIENS*}

Jean-Baptiste GOURINAT

Centre de recherches sur la pensée antique, CNRS, Paris

RÉSUMÉ. Le terme «perception » apparaît pour la première fois dans son sens philosophique dans les Académiques de Cicéron, où il traduit le terme technique stoïcien $\kappa \alpha \tau \dot{\alpha} \lambda \eta \psi(\varsigma$, traduit également par comprébension. La perception n'est pas une « perception sensible » au sens moderne du terme, car elle ne se définit pas comme une impression produite en nous par les choses extérieures, mais comme l'assentiment donné à la phantasia dite compréhensive ou perceptive, c'est-à-dire celle qui est conforme à son objet, claire et distincte. Il s'agit pour les stoïciens de l'un des critères de la vérité. Pour contester l'existence de la perception et son rôleclé dans l'épistémologie stoïcienne, les académiciens ont adopté une stratégie en deux temps: affirmer que l'assentiment porte sur des propositions et non sur des représentations, et contester l'existence d'une représentation perceptive, discernable des représentations fausses. Les stoïciens ont répondu sur ces deux points et ont reconstruit la notion de perception inventée initialement par Zénon dans le cadre de cette polémique, qui a forgé la notion classique de la perception. Le continuisme perceptif des stoïciens et leur conception cinématographique de la perception sensible sont les clés de leur conception de la perception.

SUMMARY. The word "perception" first appears in its philosophical sense in Cicero's

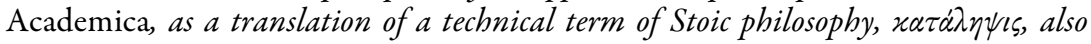
translated by comprehensio. "Perception" in its original sense is not a "sense perception" in the modern sense of the word, since it is not an impression produced in us by

* Cet article est la version remaniée d'une communication présentée au séminaire du Centre Léon Robin sur la perception, le 19 janvier 1996. Certains développements ont également fait l'objet d'une communication lors d'une journée d'étude sur Cicéron organisée par C. Grellard, D. Lefebvre et C. Lévy le 31 janvier 2011. Au cours des années, elle a bénéficié de nombreuses discussions sur cette question, notamment avec le regretté Michael Frede, avec David Sedley lors de la présentation de sa communication sur la définition stoïcienne de la phantasia kataleptike au Centre Léon Robin, et enfin avec Håvard Løkke lors d'un récent séminaire au Centre Léon Robin. 
external things, but the assent given to the so-called "perceptive" or "impressive" phantasia, i.e., to an impression conform to its object, clear and distinct. It is one of the criteria of truth of the Stoics. To challenge its key role in Stoic epistemology, the Academics adopted a two-steps strategy: they argued that the assent pertains to propositions, not to impressions, and they challenged the existence of perceptive impressions and their discernibility. The Stoics answered to these two points and rebuilt the notion initially designed by Zeno in the frame of this polemic, putting foundations for the classical notion of perception. The continuity of perception and their cinematographic conception of sense perception was one of the keys of their defence. 
Un certain nombre des termes et des concepts que nous utilisons encore sont nés dans la philosophie antique. Nous avons souvent oublié le sens qui était alors le leur, ainsi que le contexte dans lequel ils sont apparus. Ce n'est pas la moindre utilité de la lecture des philosophes antiques que de nous permettre de remonter ainsi à la source oubliée de notions que nous croyons connaitre, mais dont le sens moderne ne s'est parfois construit que par une succession de transformations à partir du sens primitif.

C'est le cas de la notion de perception. Perceptio apparaît pour la première fois dans son sens philosophique dans les Académiques de Cicéron. C'est une des trois traductions qu'il donne du grec $\kappa \alpha \tau \dot{\alpha} \lambda \eta \psi \psi i \varsigma$. Selon Cicéron, l'emploi philosophique du terme $\kappa \alpha \tau \dot{\lambda} \lambda \eta \psi$ is est lui-même une innovation terminologique et conceptuelle de Zénon de Citium. Il s'agit d'une notion centrale dans le débat épistémologique entre stoïciens et académiciens.

La question: «existe-t-il des perceptions ? » est en effet au centre de la polémique sur le critère de la vérité. Selon les classifications stoïciennes, il s'agit donc là d'une des subdivisions de la logique: dialectique, rhétorique, espèce définitionnelle, critère ${ }^{1}$. C'est en un sens une question plus centrale encore, dans la mesure où c'est elle qui détermine la ligne de partage entre dogmatisme et scepticisme. S'il y a des perceptions, on peut être dogmatique, c'est-à-dire stoïcien. S'il n'y a pas de perception, on sera sceptique, puisque l'on suspendra son assentiment.

Dans ces conditions, on ne doit pas s'étonner que le terme ait pris son essor dans les temps modernes à partir du cartésianisme et de sa réfutation du scepticisme. Le sens que le terme prend à partir de Descartes dérive assez directement de l'émergence de la notion dans le stoïcisme et de sa traduction en termes de perceptio par Cicéron. Et si Descartes utilise alternativement percipere et comprehendere dans les Méditations, c'est parce que c'est déjà ce que fait Cicéron (ainsi qu'Augustin dans le Contra Academicos)2.

1. Diogène Laërce, VII, 41.

2. Sur le fait que les Premiers Académiques de Cicéron sont une lecture de Descartes parce qu'une « référence traditionnelle dans l'enseignement des jésuites », voir Beyssade 2001, p. 29. 
C'est en mettant en parallèle les premières Méditations et les Académiques de Cicéron que l'on peut voir se former notre notion de perception, alors que trop souvent on remonte de Descartes à Aristote, ce qui n'a aucune justification historique.

Analyser l'emploi du terme chez Cicéron et, plus généralement, les polémiques entre stoïciens et académiciens dont il se fait l'écho permettra d'une part de rendre aux Académiques de Cicéron la place qui leur revient et, d'autre part, de mieux percevoir le sens originel de la notion de perception.

\section{La notion stoïcienne de perception et ses ambiguïtés

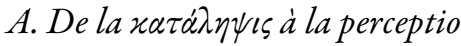

Cicéron utilise trois traductions différentes du terme $\kappa \alpha \tau \dot{\alpha} \lambda \eta \psi \iota \varsigma$ : cognitio, perceptio et comprehensio (Ac. Pr. II, 17 ; cf. De Fin. III, 17).

Comprehensio est la traduction que Cicéron qualifie à deux reprises de traduction verbum e verbo (Ac. Pr. II, 17; II, 31). Cette expression est souvent traduite par « mot à mot». Ainsi Bréhier traduit-il: « $\kappa \alpha \tau \dot{\alpha} \lambda \eta \psi(\imath$, quam ut dixi verbum e verbo exprimentes comprensionem dicemus $\gg$ (II, 31) de la manière suivante: «catalêpsis que, je l'ai dit, je traduis mot à mot par compréhension $\gg^{3}$. En fait, Cicéron veut dire plus précisément qu'il traduit le mot à partir du mot, c'est-à-dire qu'il traduit le mot à partir de son sens non philosophique d'origine par le mot latin correspondant dans le même sens non philosophique. Comprehensio traduit en effet littéralement l'idée de «saisie avec le poing » qui est celui du terme grec $\kappa \alpha \tau \dot{\alpha} \lambda \eta \psi i \varsigma$ à l'origine, et que Zénon lui conservait métaphoriquement :

Une fois qu'elle était acceptée et approuvée, il l'appelait « compréhension $\gg$, par ressemblance avec les choses que l'on prend avec la main : c'est de là qu'il avait tiré ce nom, que personne n'avait employé avant lui pour désigner une telle chose. (Ac. Post. I, 41.)

La traduction de $\kappa \alpha \tau \dot{\alpha} \lambda \eta \psi$ is par comprehensio reprend donc dans la langue latine un usage métaphorique que Zénon avait introduit dans la langue grecque pour le terme $\kappa \alpha \tau \dot{\alpha} \lambda \eta \psi i \varsigma$.

L'emploi de la traduction par perceptio repose sur la formation analogique de ce terme latin et du terme grec. Per-ceptio est formé en latin de la même manière que $\kappa \alpha \tau \dot{\alpha}-\lambda \eta \psi$ is en grec, per-pouvant être considéré comme une traduction de $\kappa \alpha \tau \dot{\alpha}$ - et -ceptio comme une traduction de $-\lambda \eta \psi i s$.

3. Bréhier 1962, p. 202. Brittain 2006, p. 20 et Kany-Turpin 2010, p. 155, traduisent par «litteraly» ou « littéralement », ce qui est encore moins précis. Les trois traducteurs traduisent de la même manière qu'en II, 31 l'occurrence de verbum e verbo en II, 17. 
Cicéron a donc pu considérer que perceptio constituait un décalque littéral de $\kappa \alpha \tau \dot{\alpha} \lambda \eta \psi \iota \varsigma$. En revanche, le sens de «saisie avec le poing» n'est pas conservé, perceptio signifiant plutôt « récolte», ce qui demeure évidemment présent dans le sens que nous employons le plus couramment en français, où la « perception » désigne le service chargé de la collecte des impôts pour le Trésor public. Le sens habituel de perceptio en latin est donc plus éloigné de celui du grec $\kappa \alpha \tau \dot{\alpha} \lambda \eta \psi \iota \varsigma$ que le terme comprehensio.

Cognitio enfin traduit plus simplement le sens dans lequel Zénon emploie le terme $\kappa \alpha \tau \dot{\alpha} \lambda \eta \psi i \varsigma$, autrement dit le sens littéral et non métaphorique que Zénon a donné au terme grec. Cette traduction est néanmoins imprécise, car elle n'indique pas quel type de connaissance est la $\kappa \alpha \tau \dot{\alpha} \lambda \eta \psi(\varsigma$, alors qu'il est évident qu'il s'agit d'une cognition d'un genre particulier.

Les trois traductions adoptées par Cicéron sont donc complémentaires: cognitio traduit le sens littéral non métaphorique, mais de façon imprécise, comprehensio et perceptio précisent le sens de la cognitio, l'un en traduisant la métaphore, l'autre par une analogie morphologique. Cicéron privilégiera comprehensio et perceptio mais il manifeste ainsi un sens remarquable du non-recouvrement des champs sémantiques de deux langues différentes: c'est ce sentiment qui explique son besoin de passer d'une traduction à l'autre.

L'usage métaphorique du terme $\kappa \alpha \tau \dot{\alpha} \lambda \eta \psi$ is fait partie d'une métaphore plus vaste qui assimile les différents états cognitifs de l'âme aux différentes positions de la main touchant ou saisissant un objet, et c'est cette métaphore développée qui donne le sens précis de ce qu'est la perception. Toujours selon Cicéron, mais cette fois dans la première version des Académiques, le Lucullus, Zénon distinguait nettement la représentation ${ }^{4}$, l'assentiment, la perception et la science en tant que quatre positions ou états différents de l'âme, comparables respectivement à la main ouverte, la main repliée, le poing fermé et le poing enserré dans l'autre main :

Zénon l'exprimait par un geste, car quand il avait montré sa main ouverte, les doigts étendus, il disait: «voilà comment est la représentation ». Quand il avait contracté légèrement ses doigts, il disait que l'assentiment est de ce genre. Puis quand il avait complètement refermé sa main et formé le poing, il disait que c'était la compréhension, et c'est à partir de cette

4. C'est ainsi que je traduirai, de manière purement conventionnelle, le terme grec $\phi \alpha \nu \tau \alpha \sigma i \alpha$ : en principe, ce terme ne désigne pas à proprement parler une «représentation », puisqu'il désigne dans la plupart des cas une impression de l'objet dans l'âme, selon la définition inaugurée par Zénon et empruntée au Théétète (Sextus Empiricus, $M$. VII, $236=S V F$ I 58 ; voir plus bas, p. 53 et 83-84). Il s'agit donc à proprement parler d'une « présentation » plutôt que d'une re-présentation. 
comparaison qu'il a donné son nom à la chose, d'un nom qui n'existait pas auparavant, $\kappa \alpha \tau \dot{\alpha} \lambda \eta \psi \mid \zeta$; enfin, quand il avait placé sa main gauche sur la droite et qu'il avait enserré son poing étroitement et fortement, il disait que telle était la science, dont personne n'est capable, sinon le sage ${ }^{5}$.

Main ouverte, main repliée, poing fermé et mains refermées l'une sur l'autre sont quatre positions différentes de la main, quatre façons de toucher et de saisir un objet. L'usage de cette métaphore de la main et du poing par les stoïciens est attesté de façon indépendante par Sextus Empiricus, qui ne l'attribue pas à Zénon, mais aux stoïciens en général, tout en la limitant à la science: la science, dit-il par deux fois, est selon les stoïciens « la partie directrice de l'âme disposée d'une certaine manière, comme le poing est la main dans une certaine position (pos echon) $»^{6}$. Même si Sextus ne l'applique qu'à la science, la métaphore se comprend dans le contexte de l'application des différentes positions de la main aux différents états cognitifs de l'âme, et l'on ne peut donc guère douter qu'il ne s'agisse de la même métaphore, que certains successeurs stoïciens de Zénon ont complétée par la notion technique de pos echon. En rapprochant la métaphore originale de Zénon exposée dans les Premiers Académiques, II, 145 de sa trace dans les textes de Sextus ( $P$. II, $81 ; M$. VII, 39) qui comparent la science au poing fermé et serré, on peut supposer que chez les successeurs stoïciens de Zénon qui ont conservé la métaphore de la main, la perception devait être, au même titre que la science, un certain état de l'âme, l'âme $\pi \omega s$ Éxov, c'est-à-dire l'âme «disposée d'une certaine manière $»^{7}$. Dans la doctrine stoïcienne, un tel état est caractérisé par sa non-permanence, par opposition à une qualification durable (celle qui résulte d'une qualité): il s'agit d'une position ou d'une disposition, comme la position assise $e^{8}$, le poing ouvert ou fermé. Il semble que les mouvements soient aussi des pos echonta, mais sur ce point nos sources sont malheureusement moins claires, car Plotin est muet sur cette question, tandis que Simplicius donne « celui

5. Cicéron, Ac. Pr. II, 145.

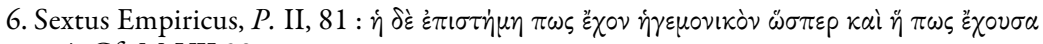

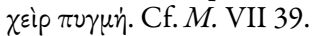

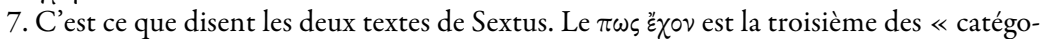
ries » ou « genres » stoïciens, répertoriée comme telle par Plotin et les commentateurs néoplatoniciens. Sur les quatre genres, voir notamment Plotin, Enn. VI, 1 [42], 25, 1 -7 ; Simplicius, In Categ. 66, 32-67, 22 Kalbfleisch. Sur le $\pi \omega \varsigma$ हैxov, voir Alexandre, In Top. 360, 9-13 Wallies; Plotin, Enn. VI, 1 [42], 30, 1-27 ; Simplicius, In Categ. 67, 2-8 ; 173, 24-28 ; 212, 7-213, $1 \mathrm{~K}$. Pour une présentation de la doctrine des quatre genres, voir Long-Sedley 1987 , 27-29, t. I, p. 162-179.

8. Simplicius, In Categ. 67, $4 \mathrm{~K}$.

9. En dehors des deux passages de Sextus cités n. 6 supra, voir Alexandre d'Aphrodise, In Topic. 360, 9-13 W. 
qui court » comme exemple de l'un des sens de la catégorie du «quel $\gg^{10}$, sens qui précisément semble correspondre au pos echon, mais sans que cela soit explicite ${ }^{11}$. En tout état de cause, l'assentiment, comme la représentation et l'impulsion, sont des mouvements de l'âme ${ }^{12}$, de sorte qu'ils sont bien l'âme pos echon, ce qui implique que, au moins en ce qui concerne l'âme, certains mouvements relèvent bien du pos echon. Les modifications qui consistent dans l'âme pos echon, malgré l'image statique de la main dans une certaine position, sont donc moins des états que des mouvements. Mais, dans certains cas, toutefois, la disposition peut devenir durable : c'est le cas de la science, qui sera décrite comme une disposition ferme et inébranlable, et l'on peut penser qu'il en va de même pour la compréhension: de fait, Plutarque, qui décrit représentation, assentiment et impulsion comme des mouvements, n'y inclut pas la compréhension - mais cela peut être dû soit au contexte, soit au fait qu'il considère la compréhension comme une espèce de l'assentiment. La comparaison entre les différents états de la main et les différentes dispositions cognitives de l'âme (représentation, assentiment, perception, science) est précisément utilisée pour décrire des degrés différents de fermeté et de stabilité dans des processus qui vont du simple toucher à la saisie ferme. Dans la métaphore développée par Zénon telle qu'en témoigne Cicéron, de même que le poing est la main dans une certaine position, la perception est l'âme dans une certaine position, mais ces positions sont loin d'être identiques, et il semble que certaines de ces «positions » soient en fait des mouvements, tandis que d'autres ont plus de stabilité. Une complication supplémentaire vient du fait que certains au moins de ces mouvements de l'âme ne sont pas seulement des mouvements de l'âme, mais sont aussi des facultés $\left(\delta \nu \nu \dot{\alpha} \mu \varepsilon 1 \varsigma^{13}\right)$.

10. Simplicius, In Categ. 217, 16-18 K.

11. Simplicius explique qu'il y a trois sens du « quel », dont certains durables, d'autres non, et un seul qui correspond à la qualité. Sur l'articulation complexe des catégories entre elles, voir Duhot 1991.

12. Plutarque, $A d v$. Colotem, 26, 1122 B.

13. Le témoignage du De anima de Jamblique, cité par Stobée, Eclog. I, 49, 34, p. 369, 6 -

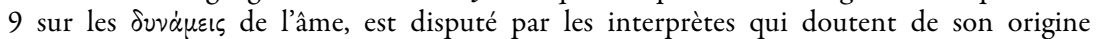
stoïcienne : le plus radical sur ce point est Tieleman 2003, p. 38 ; voir aussi la discussion de Goulet-Cazé 2011, p. 80-89, avec de nombreuses références aux discussions antérieures (sauf Tieleman) mais aucune conclusion définie. Or, pour une raison qui m'échappe, les interprètes qui ont discuté l'authenticité stoïcienne du terme ne tiennent pas compte du fait qu'il

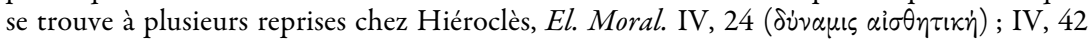

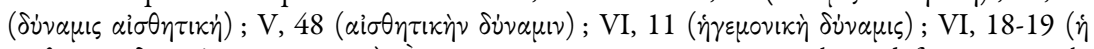

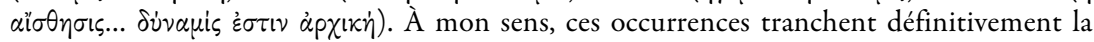
question et ne peuvent laisser de doute sur l'authenticité du terme, au moins en ce qui concerne la «faculté sensitive » et la « faculté directrice » de l'âme. 
Entre la représentation, l'assentiment et la perception, le texte du Lucullus II, 145 fait seulement état d'une différence de position ou de disposition de l'âme, comparable à différentes positions de la main, différentes façons de saisir un objet, selon une fermeté croissante. Mais, de fait, la description des Académiques I, 41 implique une différence plus radicale entre une pure passivité qui est celle de la représentation et un acte d'approbation et d'acceptation qui commence avec l'assentiment. La représentation consiste simplement à être affecté et modifié par une chose extérieure, à en recevoir une impression, tandis que l'assentiment consiste en une manière d'approbation, une façon d'《 ajouter foi » à la représentation, selon l'expression de Cicéron ${ }^{14}$. C'est donc un acte d'adhésion de l'âme à l'impression qu'elle reçoit, tandis que la représentation est un phénomène passif. C'est ce que la métaphore dissimule quelque peu, mais que confirme Sextus, $M$. VII, 237, selon qui la perception est, au même titre que l'assentiment et l'impulsion, une modification de l'âme différente de la représentation, qui est passive, tandis que la perception et les autres modifications

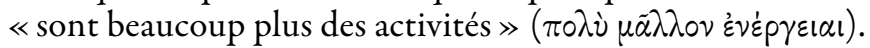

Dans la séquence graduée décrite dans le Lucullus II, 145, la perception apparaît comme distincte de l'assentiment, de sorte que l'assentiment apparaît comme l'état intermédiaire entre représentation et perception. Mais il y a en fait deux sens du terme « assentiment » : un sens spécifique et un sens générique $^{15}$ qui permet de définir tout aussi bien la perception ou compréhension comme une forme spécifique d'assentiment. C'est la définition que transmet Sextus, rapportant la polémique d'Arcésilas contre Zénon : $\dot{\eta}$

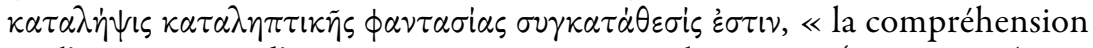
est l'assentiment d'une représentation compréhensive $\gg\left(M\right.$. VII 154); $\eta^{\tilde{\nu}}$ $\kappa \alpha \tau \alpha \lambda \eta \pi \tau \iota \kappa \tilde{n} \phi \alpha \nu \tau \alpha \sigma i \alpha, \sigma \nu \gamma \kappa \alpha \tau \dot{\alpha} \theta \varepsilon \sigma \iota$, , « c'est l'assentiment à une représentation compréhensive $\gg\left(M\right.$. VII, $\left.155^{16}\right)$.

14. Cicéron, Ac. Post. I, 41 : adiungebat fidem.

15. C'est déjà ce qu'avait remarqué Kerferd 1978, p. 255.

16. À quelques lignes de distance, Sextus parle d'《 assentiment d'une représentation », au génitif ( $\kappa \alpha \tau \alpha \lambda \eta \pi \tau i k \eta \tilde{s} \phi \alpha \nu \tau \alpha \sigma i \alpha \varsigma)$, puis $\mathrm{d}^{\prime} \ll$ assentiment à une représentation », au datif ( $\alpha \tau \tau \alpha \lambda \eta \pi \tau \kappa \kappa \tilde{n} \phi \alpha \nu \tau \alpha \sigma i \alpha$ : M. VII, 153). D'après ce qu'il dit dans le reste du passage, il apparaît toutefois avec une certaine évidence que l'assentiment n'est pas pour les stoïciens l'opération effectuée par une représentation (ce que pourrait laisser penser la construction avec le génitif), mais l'opération qui porte sur une représentation, puisque, en VII, 154, Sextus conteste aux stoïciens que l'assentiment soit « relatif à une représentation », $\pi$ pòs $\phi \alpha \nu \tau \alpha \sigma i \alpha \nu$. On doit toutefois remarquer qu'il y a selon Cicéron une quasi-acceptation par les sens («quasi accepta sensibus 》) qui est différente de l'assentiment, puisque l'assentiment s'y ajoute (Ac. Post. I, 40). Mais le quasi marque précisément une nuance. La faculté et l'acte d'assentiment sont bien différents de la représentation et ne sont pas effectués par cette dernière. Voir plus bas la question de l'assentiment à la représentation ou à la proposition. Peut-être cette quasi-acceptation par les sens, différente de l'assentiment, peut-elle être 
Cette définition peut être à juste titre rapprochée de la description de Cicéron, qui dit que, «quand» la représentation compréhensive est 《reçue et approuvée », Zénon l'appelle « compréhension ${ }^{17}$. Dans ce sens, la compréhension est une forme de l'assentiment, qui doit être distinguée d'une autre forme d'assentiment plus faible, qui est l'opinion, définie comme assentiment faible ou assentiment à ce qui n'est pas perçu. L'opinion ainsi définie correspond alors à l'assentiment pris dans le sens spécifique du terme dans le passage du Lucullus I, 145. C'est la position attribuée par le même Cicéron à Zénon dans les Seconds Académiques, II, 42, où la compréhension apparaît comme un intermédiaire entre la science et l'ignorance, qui est décrite alors par Cicéron comme la source de l'opinion. De fait, l'opinion est généralement définie par les stoïciens comme $\sigma v \gamma$ $\kappa \alpha \tau \dot{\alpha} \theta \varepsilon \sigma \iota \varsigma \dot{\alpha} \sigma \theta \dot{\varepsilon} \nu \eta \varsigma$, ce que Cicéron traduit par imbecilla adsensio ${ }^{18}$. Si l'on compare les deux textes de Cicéron et le texte parallèle de Sextus, $M$. VII, 151-152, on constate que la katalepsis occupe toujours une position intermédiaire et que la science est toujours le terme dernier de la hiérarchie. Mais le terme qui précède la katalepsis et par rapport auquel celle-ci se trouve en position médiane varie d'un texte à l'autre : dans le Lucullus II, 145, c'est l'assentiment qui précède (mais Cicéron ne dit pas en l'occurrence que la compréhension est intermédiaire, il se contente de la mentionner après) ; dans la seconde version des Académiques I, 41, la compréhension est intermédiaire entre la science et l'ignorance (inter scientiam et inscientiam), qui est la source de l'opinion (ex qua existebat etiam opinio); chez Sextus, M. VII, 151-152, la katalepsis est intermédiaire entre la doxa et la science. Sextus dit une fois que la katalepsis est « à la frontière entre les

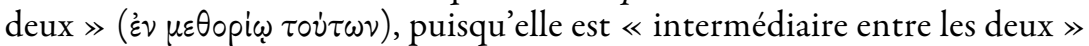
( $\tau \grave{\eta} \nu \mu \varepsilon \tau \alpha \xi \dot{\nu} \tau o \nu ่ \tau \omega \nu)$. La première des deux expressions pourrait avoir un sens légèrement dépréciatif mais elle pourrait aussi dériver de la source sceptique de Sextus ${ }^{19}$, de sorte qu'il serait sans doute imprudent d'en tirer trop de conclusions quant à la doctrine stoïcienne. En tout état de cause, dans les trois témoignages, la katalepsis occupe une place intermédiaire entre un assentiment inébranlable et un assentiment qui est soit indéterminé, soit faible ou faux, identifiable à l'opinion ou source de celle-ci ${ }^{20}$.

assimilée à ce que les stoïciens appellent l'घí̌̆ls (voir notamment Plutarque, Virt. Moral. 7, 447 A ; Diogène Laërce, VII, 51, et la discussion de Goulet-Cazé 2011, p. 121-174).

17. Cicéron, Ac. Post. I, 41. Voir Goulet-Cazé 2011, p. 195.

18. Comparer Sextus, M. VII, 151 et Cicéron, Ac. Post. I, 42.

19. Voir sur ce point Ioppolo 2009, p. 87.

20. Pour une comparaison entre Académiques, I, 41 et Sextus, M. VII, 151-152, voir notamment Ioppolo 1986, p. 21-28, Ioppolo 2009, p. 84-91, Goulet-Cazé 2011, p. 188-199. 
Quant à la sensation ( $\alpha$ l゙ $\theta \eta \sigma \iota s$, sensus), elle sera elle-même une forme de compréhension ou perception. Zénon aurait en effet considéré la sensation comme la combinaison d'une représentation et d'un assentiment (Ac. Post. I, 40). Mais le terme « sensation » est ambivalent, car quod erat sensu comprensum, id ipsum sensum appellabat («ce qui était compris par un sens, il

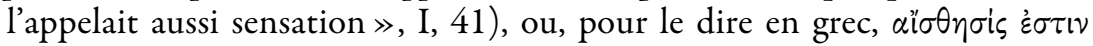
$\dot{\alpha} \nu \tau i \lambda \eta \psi i s<\delta l^{\prime}>\alpha i \sigma \theta \eta \tau \eta p i o v \dot{\eta} \kappa \alpha \tau \dot{\alpha} \lambda \eta \psi i s$, « la sensation est soit une saisie par un organe des sens, soit une compréhension $\gg^{21}$. Autrement dit, il y a un sens de « sensation » qui désigne un simple affect sensoriel, une aperception par un organe sensoriel. Mais, dans un autre sens, la sensation, c'est une perception, c'est-à-dire la combinaison d'une représentation sensorielle perceptive et de l'assentiment donné à cette représentation.

On a donc l'ensemble suivant :

- la représentation ( $\phi \alpha \nu \tau \alpha \sigma i \alpha, v i s u m)$ est comme un impact ou une impression venue de l'extérieur (Ac. Post. I, 40), équivalente à la main ouverte qui effleure les choses du bout des doigts (Ac. Pr. II, 145).

- l'assentiment ( $\sigma 0 \gamma \kappa \alpha \tau \dot{\alpha} \theta \varepsilon \sigma \iota$, , adsensio, approbatio) prend soit une forme faible, visant une représentation non perçue - il s'agit alors d'une opinion ( $\delta \dot{o} \xi \alpha$, opinio), comparable aux doigts contractés -, soit une forme forte, visant une représentation perceptive - il s'agit alors d'une perception

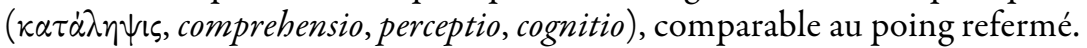
- la science ( $\dot{\pi} \tau \sigma \tau \eta \dot{\mu}$, scientia) est considérée tantôt comme un assentiment ferme et irréversible, à la manière de la main gauche appliquée sur le

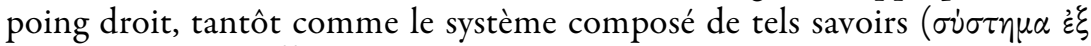
$\left.\dot{\varepsilon} \pi \iota \tau \tau \eta \mu \tilde{\omega} \nu \tau 0 เ ૦ \dot{\tau} \tau \omega \nu^{22}\right)$.

\section{B. Les ambigü̈tés de la notion de perception}

Qu'est-ce que saisit la perception? Une chose? Une proposition ? Une représentation? Il y a sur ce point un flottement et une ambiguïé, voire déjà des éléments de polémique.

L'image de la main semble indiquer que c'est la chose elle-même qui est représentée, acceptée, perçue et connue : toutes ces opérations apparaissent comme autant d'états successifs de l'âme se refermant progressivement sur son objet.

21. [Plutarque], Opinions des philosophes, IV, 8, 1, 899 D. Le latin antique ne connaît

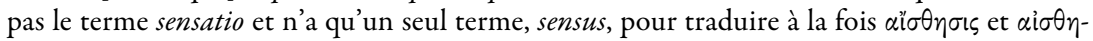

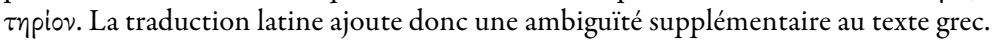

22. Stobée, Eclog. II, 7, p. 73, 19-74, 3 W. (SVF II 112). Pour un commentaire de ce texte et l'analyse de la notion de « science », voir Gourinat 2011. 
De fait, le modèle du toucher n'est pas seulement une métaphore. Pour les stoïciens, la représentation n'est pas une image ${ }^{23}$ : ils refusent la conception épicurienne selon laquelle il y aurait dans notre âme des images des choses, les fameux «simulacres ». Pour Zénon, la représentation est une impression $^{24}$, ce n'est pas une image mentale par reflet ou projection. Il y a une forme de contact de la chose à l'âme, qui, en quelque sorte, touche la chose: même dans la vision oculaire, il y a des espèces de bâtons ${ }^{25}$ qui sont comme les doigts de la main dans la métaphore de Zénon. Précisément pour cette raison, il s'agit d'autre chose que d'une simple métaphore. Bien sûr, le terme même d' «impression » est une métaphore ${ }^{26}$, car la réalité psychique n'est pas un bloc de cire dans lequel il puisse y avoir de véritable impression. Mais la métaphore fonctionne en l'occurrence plutôt comme un modèle. Le processus est exactement analogue.

Dans ces conditions, il semble bien que ce soit la chose même qui soit l'objet de la perception. De fait, Cicéron emploie souvent les expressions rem percipere («percevoir une chose») ou res percipere («percevoir des choses ») : Ac. Pr. II, 26; 44; 101; Ac. Post. I, 31.

Pourtant, d'après d'autres textes, il semble plutôt que ce soient des propositions ou des représentations qui sont l'objet de l'assentiment, et donc de la perception. Stobée, Ecl. II, 9, p. 88, 4 (SVF III 171), citant probable-

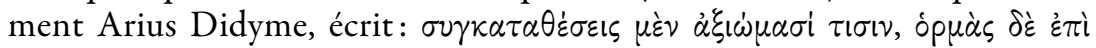
$\kappa \alpha \tau \eta \gamma \circ p \eta \mu \alpha \tau \alpha$, «les assentiments portent sur certaines propositions, les impulsions sur des catégorèmes... »

Pour Brad Inwood, il s'agirait là de la véritable doctrine stoïcienne, parce qu'elle constitue une version moins simplifiée et plus «précise » que la version de l'assentiment à la représentation ${ }^{27}$. Si on l'interprète comme un argument ad hominem (c'est-à-dire comme un argument qui reprend une thèse stoïcienne pour la retourner contre les stoïciens), l'argument d'Arcésilas rapporté par Sextus, $M$. VII, 154, paraît aller dans le même sens:

Si précisément la perception est l'assentiment d'une représentation perceptive, elle est inexistante, d'abord parce que l'assentiment n'est pas relatif à la représentation mais au langage (car les assentiments portent sur les propositions), et deuxièmement parce qu'on ne trouve aucune représen-

23. Gourinat 1996, p. 36-38.

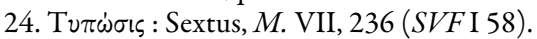

25. Cf. Alexandre d'Aphrodise, De mantissa, 130, 14 Bruns (SVF II 864); Galien, Plac. Hipp. et plat. VII, 7, 21 (SVF II 865) : il s'agit de l'air tendu en forme de cône parce qu'il est « piqué » par le pneuma de l'œil décrit par [Plutarque], Plac. IV, 15, 901 D-E (SVF II 866).

26. Alexandre d'Aphrodise, De anima, 72, 11-13 Bruns (SVF II 58).

27. Inwood 1985, p. 56-57. Dans le même sens déjà : Voelke 1973, p. 30. 
tation vraie qui ne serait pas susceptible de devenir une représentation fausse, comme c'est établi par de nombreux exemples variés.

Arcésilas paraît ici tout simplement rappeler à Zénon qu'il ne peut pas y avoir de perception puisqu'il définit la perception comme un assentiment portant sur une représentation perceptive alors que la doctrine de Zénon est bien par ailleurs que l'assentiment porte sur une proposition, et non pas sur une représentation : la notion d'assentiment à une représentation - fût-ce une représentation perceptive - est alors inconsistante.

La thèse d'un assentiment à la proposition semble d'ailleurs parfaitement illustrée par un passage de Cicéron, selon qui ea quae... percipi dicuntur, « ces choses dont on dit qu'elles sont perçues », c'est : illud est album, hoc dulce, canorum illud, hoc bene olens, hoc asperum, «ceci est blanc, cela est doux, ceci, c'est mélodieux, cela sent bon, et cela, c'est rugueux » ( $A c$. $\operatorname{Pr}$. II, 21). Autrement dit, à première vue, ces choses perçues prennent la forme de propositions.

Il est clair cependant qu'en réalité la notion de $\kappa \alpha \tau \dot{\alpha} \lambda \eta \psi i s$ (perception)

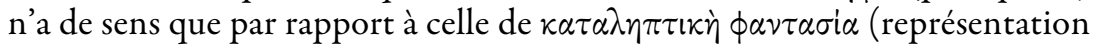
perceptive). Aussi Cicéron dit-il très souvent que « ce qui est perçu », c'est une représentation, visum. Ainsi en Ac. Pr. II, 77 :

- Qu'est-ce qui peut être perçu ?

- Une représentation, je crois.

Beaucoup d'autres textes disent la même chose (Ac. Post. I, 41; Ac. Pr. II, $40 ; 41 ; 99)$. S'il en est ainsi et si la perception est bien une forme de l'assentiment, alors l'objet de l'assentiment et de la perception est bien une représentation. Pour autant, une telle représentation n'est pas nécessairement d'origine sensorielle. L'ensemble des développements de Cicéron dans les Premiers Académiques, ainsi qu'un texte très éclairant de Galien ${ }^{28}$, indiquent assez clairement que les représentations perceptives qui peuvent faire l'objet de perceptions ne sont pas seulement des représentations sensorielles. Ainsi le texte de Galien explique-t-il qu'un théorème des Éléments d'Euclide peut faire l'objet d'une perception ${ }^{29}$. Nous dirions

28. Galien, De animi peccatis, p. 58 Kühn (SVF III 172). Texte, traduction et commentaire dans Goulet-Cazé 2011, p. 219-236. Voir déjà Gourinat 1996, p. 77.

29. Il faut reconnaître qu'il n'est pas certain que l'exemple vienne des stoïciens, qui du reste ne sont pas cités explicitement par Galien. Mais plusieurs témoignages antiques attribuent aux stoïciens l'existence d'une compréhension (katalepsis) résultant d'une démonstration (Cicéron, $A c$. Pr. II, 26 ; Diogène Laërce, VII, 45 ; VII, 52) ou d'un raisonnement (Anonyme de Turin, X, Fol. 92v, 12-14). Par exemple, qu'il existe des dieux et qu'ils sont providentiels, c'est ce dont j'ai la perception $\left(\kappa \alpha \tau \dot{\alpha} \lambda \eta \psi(\varsigma)\right.$ grâce à une démonstration, $\delta \iota^{\prime}$ $\dot{\alpha} \pi \circ \delta \varepsilon \dot{\xi} \xi \omega \varsigma$ (Diogène Laërce, VII, 52). Et comme le fait remarquer Sedley 2005, p. 91, « on voit mal comment une katalepsis non sensorielle pourrait être un assentiment à autre chose 
plutôt, en prenant l'autre traduction de Cicéron, que nous pouvons le comprendre ou non. La double traduction de Cicéron par les deux termes «compréhension » et «perception » a donné lieu à deux notions différentes, mais la notion zénonienne inclut les deux notions - la perception des choses sensibles et la compréhension d'objets que nous ne comprenons que par des raisonnements. En revanche, pour les stoïciens - peut-être s'agit-il là d'une doctrine postérieure à Zénon, mais elle n'en est pas moins stoïcienne $e^{30}-$, comprendre un théorème d'Euclide et percevoir que ceci est blanc, c'est la même opération psychique, celle qui consiste à donner son assentiment à une représentation perceptive de la chose, même si l'origine de la représentation n'est pas la même. Or, l'exemple du théorème mathématique montre très bien pourquoi la notion de perception ou de compréhension n'a de sens que par rapport à la notion de représentation perceptive. En ce qui concerne le contenu propositionnel, le géomètre et le néophyte se représentent exactement la même chose. Mais le géomètre, parce qu'il connaît l'ensemble des Éléments, comprend vraiment la proposition, de la même manière que, selon Galien, un profane en mathématique comprend que deux et deux font quatre ${ }^{31}$ : il serait capable de démontrer ce théorème grâce à sa maîtrise des Éléments. Un profane n'a pas de connaissance en mathématiques, et, à la même proposition, il ne donnera qu'un assentiment faible: il aura l'opinion que le théorème est vrai par exemple parce que le géomètre lui dira qu'il est vrai. Un ignorant ne peut donc pas comprendre le théorème parce qu'il n'en a pas de représentation compréhensive. Pourtant, il se représente la même proposition que le géomètre. Mais son assentiment ne peut pas être le même, il ne peut être que faible, parce qu'il est assentiment à quelque chose qui n'a pas été perçu. Il est clair dès lors que la notion de perception n'a de sens que par rapport à la représentation que nous nous faisons de la proposition ${ }^{32}$.

qu'une phantasia elle-même non sensorielle ». Voir Frede 1999, p. 298, qui recommande d'éviter the rash, and wrong assumption that all cases of cognition are cases of perception in our sense, even though cases of perceptual cognition are paradigms of cognition [...] The Stoics surely do not mean to say that we know the theorems of a science, for instance geometry, as a matter of perceptual cognition.

30. Sedley 2005, p. 91-92, pense que cette extension de la perception à des représentations non sensorielles, dépassant le modèle du Théétète, doit être attribuée à Zénon luimême, car il n'avait pas d'autre critère de vérité que la perception. C'est plausible, mais non certain.

31. Cf. Goulet-Cazé 2011, p. 224-225.

32. Voir plus bas, p. 67-69, la difficulté particulière soulevée par deux représentations concurrentes d'un même objet: « cette femme est Hélène » et «il n'est pas incroyable que cette femme soit un fantôme, et non pas Hélène ». Chacune a un contenu propositionnel propre, mais elles proviennent du même objet. 
On notera finalement que la proposition est le contenu de la représentation, parce qu'elle est un exprimable, c'est-à-dire ce qui est parallèle et

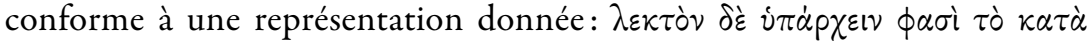

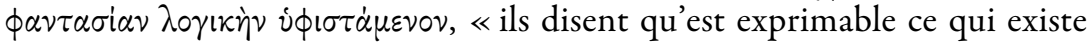
dans une représentation rationnelle » (Sextus, M. VIII, 70). L'alternative entre l'assentiment à la représentation et l'assentiment à la proposition n'en est donc pas vraiment une : il n'y a pas de représentation sans contenu, ni de contenu propositionnel auquel on puisse donner son assentiment si on ne se le représente pas d'une façon ou d'une autre. Toute représentation rationnelle est susceptible d'être exprimée dans le langage et possède un contenu propositionnel. Tout assentiment doit donc être en un sens assentiment à une représentation et en un sens assentiment à une proposition. Mais la proposition est ce qui est seulement sous-jacent dans un certain type de représentation, ce qui en est exprimable. En ce sens, la notion de perception ne peut se comprendre que par référence à la qualité de la représentation, et non d'après la proposition qui en est le contenu. C'est donc à proprement parler à la représentation que l'on donne ou refuse son assentiment ${ }^{33}$.

Aussi est-ce en percevant que nous « saisissons » les choses. Car ce que nous percevons, ce sont des représentations, mais ce que nous nous représentons, ce sont des choses.

\section{La définition stoïcienne de la représentation perceptive}

La conséquence, c'est qu'une grande part de la polémique entre stö̈ciens et académiciens sur la perception porte en réalité sur l'existence des représentations perceptives, puisque «s'il n'y a pas de représentation perceptive, il n'y aura pas non plus de perception $\gg$ (Sextus, M. VII, 155).

C'est Sextus, $M$. VII, 248, qui nous donne une citation littérale de la définition de Zénon:

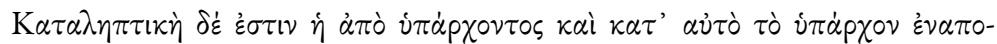

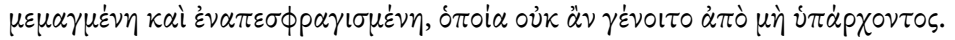

Une représentation perceptive est celle qui provient de ce qui est, qui est imprimée et marquée conformément à ce qui est, et telle qu'elle ne pourrait pas provenir de ce qui n'est pas.

Mais c'est par la traduction latine qu'en donne Cicéron dans le Lucullus que nous savons qu'il s'agit de la définition de Zénon ${ }^{34}$ :

33. Sur l'harmonisation des deux positions, voir Gourinat 1996, p. 70-71, Brennan 2005, p. 56-57, Goulet-Cazé 2011, p. 95-96.

34. Cicéron, $A c$. $\operatorname{Pr}$. II, 77. Cicéron ajoute la troisième clause de la définition quelques lignes plus bas, en l'attribuant aussi à Zénon. La définition apparaît tantôt dans la version 
Illum ita definisse: ex eo quod esset sicut esset impressum et signatum et effictum.

Il l'a définie ainsi : celle qui provient de ce qui est, qui est imprimée et marquée et figurée conformément à ce qui est.

Comme l'ont fait remarquer F. Sandbach et plus nettement encore G. Striker, cette définition n'est pas une définition qui permettrait de reconnaître subjectivement une représentation perceptive, mais sa définition d'un point de vue objectif: il s'agit d'en décrire les principales propriétés objectives, et non pas d'en permettre la reconnaissance ${ }^{35}$. De fait, les stoïciens considèrent qu'il n'y a pas besoin de définir ou de décrire la représentation perceptive ou d'en fournir des marques de reconnaissance. En effet, d'après Cicéron, $A c$. Pr. II, 17, « ils affirmaient qu'il n'était pas nécessaire de définir ce qu'est la connaissance ou la perception ou (si nous voulons un mot à mot) la compréhension qu'ils appellent $\kappa \alpha \tau \dot{\alpha} \lambda \eta \psi(\zeta)$. La représentation perceptive est en effet reconnaissable à son évidence ; or, rien n'est plus clair que l'évidence (ibid.; $c f$. Sextus, $M$. VII, 364). Cette évidence distingue en effet la représentation perceptive des autres comme les serpents « cornus » ont une marque qui les distingue des autres serpents ${ }^{36}$.

La définition est assez longuement commentée par Sextus, dans un développement qui reprend point par point les éléments de la définition de Zénon, et qui est manifestement d'origine stoïcienne. Il est à première vue assez vraisemblable que le commentaire provient de Chrysippe, dont on sait qu'il avait l'habitude de commenter les définitions de Zénon ${ }^{37}$, et qui en outre était connu pour citer très fréquemment Euripide ${ }^{38}$, dont le commentaire rapporté par Sextus cite l'Oreste. Ce n'est toutefois pas sûr, et il se peut que certains éléments remontent à Zénon ou au contraire à une déformation postérieure à Chrysippe.

\section{A. La représentation perceptive provient de ce qui existe}

Le premier point est que la représentation perceptive est « celle qui pro-

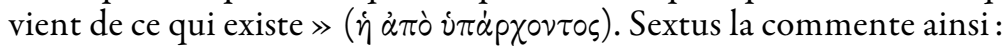

courte d'abord donnée par Cicéron (Diogène Laërce, VII, 46; Sextus, M. XI, 183; Augustin, Contr. Acad. II, 5, 11), tantôt dans la version plus longue transmise ensuite par Cicéron (Diogène Laërce, VII, 50 ; Sextus, $P$. II, 4). Sur cette définition l'analyse la plus détaillée est celle de Sedley 2005.

35. Sandbach 1971, p. 16-19; Striker 1974, p. 85-86; Striker 1990, p. 152.

36. Sextus, $M$. VII, $252 ; c f$. Cicéron, Ac. Post. I, 41. Cette évidence caractéristique des représentations perceptives rejoint malgré tout le deuxième point de la définition, c'est-àdire la précision qui distingue ces représentations. Il s'agit d'une propriété (i $\delta i \omega \mu \alpha)$ de la représentation perceptive.

37. Voir Tieleman 2003, p. 96-97.

38. Diogène Laërce, VII, 180. 
La première propriété consiste à provenir de ce qui existe, car beaucoup de représentations dérivent de choses qui n'existent pas, comme dans le cas des fous, et ces représentations ne peuvent être perceptives. ( $M$. VII, 249.)

Cette explication est en soi claire, mais, dans sa littéralité, elle n'est pas tout à fait compatible avec la doctrine de Chrysippe, et elle ne va pas sans difficulté. Selon cette explication, cette première propriété (iঠi $\omega \mu \alpha)$ de la représentation compréhensive semble renvoyer à la distinction entre représentation $(\phi \alpha \nu \tau \alpha \sigma i \alpha)$ et hallucination ( $\phi \alpha \nu \tau \alpha \sigma \tau i k o ́ v)$. Cette distinction est expliquée dans le De placitis, où elle est attribuée à Chrysippe :

Une hallucination est une attraction à vide, un affect qui arrive dans l'âme sans être produit par un objet de représentation, comme lorsque quelqu'un se bat contre une ombre ou qu'il bat des mains dans le vide. Car pour une représentation, il y a un objet susceptible de produire une représentation

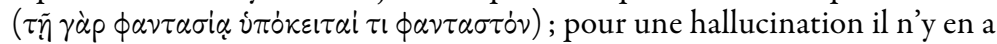
pas $^{39}$.

L'objet susceptible de produire une représentation, le « représentable » ( $\phi \alpha \tau \tau \sigma \tau \tau \dot{\delta} v)$ est en effet défini dans le même texte du De placitis comme

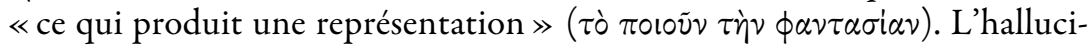
nation consiste donc à se représenter un être là où il n'y a rien ni personne: lutter contre un fantôme, ou battre des mains dans le vide, voir un monstre effrayant là où il n'y a personne. Dans la théorie stoïcienne, les représentations sensorielles proviennent des affects des organes des sens. Lorsque l'affect, par la suite d'un dérèglement, se produit sans objet, il y a dans l'âme ce que les stoïciens appellent une «attraction à vide». Les fous et les mélancoliques ont des hallucinations, mais les rêves sont également des hallucinations (Diogène Laërce, VII, 50). La clause selon laquelle la représentation perceptive doit provenir de ce qui existe semble indiquer qu'il doit s'agir d'une véritable représentation et non d'une hallucination.

Mais, dans ce cas, l'explication donnée par Sextus ne semble pas provenir directement de Chrysippe, car elle ignore précisément la distinction entre représentation et hallucination, en parlant de « représentations qui proviennent de ce qui n'existe pas », et non pas d'hallucinations. Pour que l'explication avancée par Sextus soit parfaitement cohérente avec la distinction entre représentation et hallucination, il faudrait que Sextus dise que la représentation provient de ce qui est, et non d'hallucinations qui n'ont aucun objet, et non pas qu'il dise qu'il y a beaucoup de représentations qui proviennent de ce qui n'existe pas. De fait, quelques paragraphes plus haut

39. [Plutarque], Plac. IV, 12, 900 E-F (SVF II 54). L'attribution de la doctrine à Chrysippe est confirmée par Diogène Laërce, VII, 50 (SVF II 55), qui l'attribue au livre II du traité De l'âme. 
(VII, 245), Sextus présente comme une représentation « à la fois vraie et fausse $\gg$ et non comme une hallucination la représentation qu'on a dans les rêves et qui se produit à la suite d'une « attraction à vide » : il appelle donc « représentation à la fois vraie et fausse » ce que Chrysippe appelle une hallucination et qu'il distingue en tant que telle de la représentation. Selon les distinctions opérées par Chrysippe, un affect psychique provoqué par une « attraction à vide » n'est pas une représentation, tandis que, selon l'exposé de Sextus, il s'agit d'une représentation à la fois vraie et fausse. À vrai dire, la doctrine exposée alors par Sextus paraît assez confuse, car il range sous la même catégorie de la représentation à la fois vraie et fausse deux cas distincts : celui où l'on a une représentation déformée d'un être effectivement présent (Oreste voit Électre comme une Furie) et celui où, par suite d'une attraction à vide, on voit Dion en rêve près de soi alors qu'il n'est pas là. $\mathrm{Or}$ seul le second cas est un cas d'hallucination. Sextus, $M$. VIII, 68, reprend l'exemple d'Oreste voyant des Furies et y ajoute celui d'Hercule prenant ses enfants pour ceux d'Eurysthée, et les analyse comme des représentations: les premières comme des représentations vides ${ }^{40}$, les autres comme des représentations «déformées » qui viennent bien des objets mais ne leur sont pas conformes.

Comme solution à l'incompatibilité entre l'explication de la première clause de la définition et la distinction entre représentation et hallucination, on pourrait supposer que Chrysippe entendait le terme phantasia à la fois dans un sens générique et dans un sens spécifique: dans un sens générique, le terme désignerait tout affect de l'âme, et dans le sens spécifique, il désignerait une impression causée par un objet extérieur par opposition au phantastikon causé par une attraction à vide de l'âme. On remarquera de fait que le terme pathos est le seul qui soit commun à la représentation et à l'hallucination ${ }^{41}$, mais que l'idée d'une passivité est aussi ce qui oppose la représentation aux activités de l'âme (impulsion, assentiment, perception ${ }^{42}$ ), et que, bien que ce terme soit commun aux deux contextes, aucune source ne définit l'«affect» comme un phénomène général de l'âme qui engloberait à la fois représentation et hallucination, de sorte que, dans ce sens très général, c'est bien le terme phantasia qui semble devoir être employé.

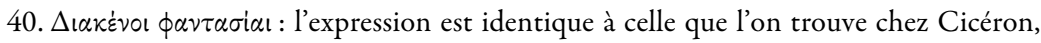
Ac. Pr. II, 49 : « images vides » (visiones inanes).

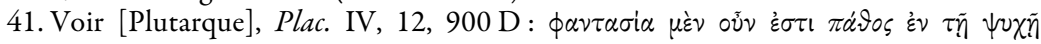

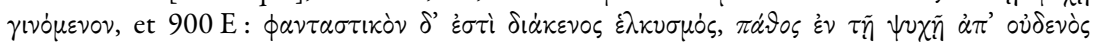

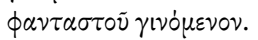

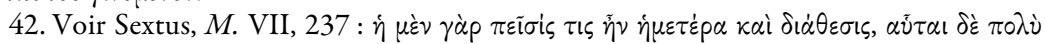

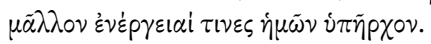


Une autre possibilité serait que cette clause de la définition de Zénon ait un tout autre sens, selon lequel la représentation «qui provient de ce qui existe » est une représentation vraie (et non pas une vraie représentation), c'est-à-dire une représentation conforme à son objet ${ }^{43}$. Une telle interprétation est suggérée par une distinction attribuée aux stoïciens, selon laquelle les représentations compréhensives font partie des représentations vraies (M. VII, 247), et c'est précisément dans ce contexte que Sextus avance et commente la définition de la représentation compréhensive ( $M$. VII, 248). Cette distinction est différente de la distinction avancée par Diogène Laërce, VII, 46, où la représentation compréhensive est directement présentée comme une espèce de la représentation. L'explication donnée par Sextus de ce qu'est une représentation vraie non compréhensive, reposant sur le cas des fous et des mélancoliques ( $M$. VII, 247), est loin d'être claire, et son exégèse de la définition reporte sur la deuxième clause la condition selon laquelle les représentations compréhensives ne sont pas vraies, en décomposant l'exégèse de la seconde clause en deux conditions distinctes, à savoir d'être vraie et d'être exacte et précise ${ }^{44}$. La décomposition de la seconde clause en deux éléments distincts d'exégèse pourrait laisser penser que, à l'origine, la première clause pourrait avoir été celle qui incluait l'exigence que la représentation soit vraie. De fait, si la décomposition exégétique rapportée ou opérée par Sextus peut sembler quelque peu artificielle, c'est peut-être parce que la définition originelle de Zénon, ne distinguant pas clairement entre une représentation déréglée et une hallucination

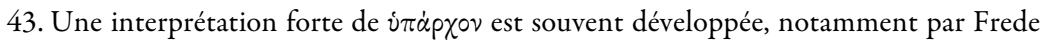
1987, p. 164-165 et Frede 1999, p. 302-304. Selon cette interprétation, il faut interpréter l'expression dans le sens technique développé par les stoïciens, dans lequel le vrai est défini comme i்ḋpxov (Sextus, M. VIII, 10). La définition signifierait alors que la représentation compréhensive provient de ce qui est vrai, sens que tend à donner Cicéron, par exemple en Ac. Pr. II, 112. « Dans ce cas, le point de la première clause ne serait pas que l'impression cognitive a son origine dans un objet réel, mais dans un fait » (Frede 1999, p. 302). Frede tire notamment argument de la critique de Carnéade rapportée par Sextus, M. VII, 402, où l'exemple d'Héraclès prenant ses enfants pour ceux d'Eurysthée est traité comme une repré-

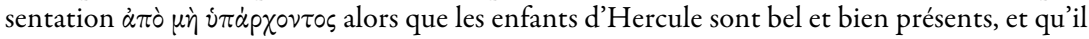
ne s'agit pas d'une hallucination. Mais il est loin d'être sûr que cette critique de Carnéade rapporte correctement ce que soutenait Zénon, car Sextus, $M$. VIII, 67, rapportant la position « des stoïciens », dit qu'Hercule a des représentations « déformées », qui sont $\dot{\alpha} \pi \grave{~}$

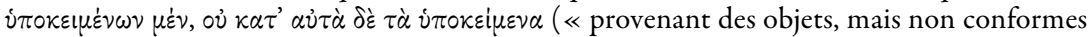

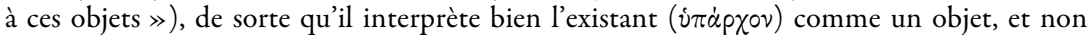
comme une proposition ou un fait. Il n'empêche, comme je vais l'indiquer, que Zénon pourrait bien avoir pris l'expression $\dot{\alpha} \pi \grave{o}$ i $\pi \dot{\alpha} p x \circ v \tau o \varsigma$ comme se référant à l'objet tel qu'il est, et non pas seulement à l'existence d'un objet. Il me semble donc que Frede 1999, p. 303, a raison de dire que cette clause de la définition contient une « ambiguïté, se référant soit à un objet soit à un fait portant sur cet objet ».

44. Sextus, $M$. VII, 249-251; voir ci-dessous, section (B), p. 63-65. 
comme le fera Chrysippe, incluait dans la première clause les représentations qui ne correspondent pas à leur objet réel, soit que cet objet n'existe pas, soit qu'il soit vu de manière déformée et pris pour ce qu'il n'est pas.

Ce qui peut expliquer le plus naturellement l'absence du recours à la distinction entre représentation et hallucination, c'est aussi le fait que l'exégèse reprend les termes mêmes de la définition de Zénon. Or il est suffisamment attesté que c'est Chrysippe qui a imposé la distinction entre représentation et hallucination pour que l'on puisse être à peu près assuré que Zénon n'a jamais fait cette distinction et que, de son côté, il a considéré les hallucinations comme des représentations. S’il en est bien ainsi, les représentations à partir de ce qui n'est pas pouvaient pour lui désigner aussi bien ce que Chrysippe appellera des hallucinations que ce qu'il considérera comme des représentations à la fois vraies et fausses : une trace de cette indistinction paraît en effet exister dans l'exposé de Sextus, M. VII, 249, lorsqu'il range sous la même catégorie de la représentation à la fois vraie et fausse à la fois d'authentiques représentations qui ne font que représenter leur objet de façon erronée (Électre qu'Oreste voit comme une Furie) et des «attractions à vide» produites dans les rêves. C'est seulement lorsque Chrysippe a distingué les hallucinations des représentations qu'a pu s'opérer l'exégèse dans laquelle c'est dans la première clause de la définition que l'on dit que la représentation perceptive n'est pas une hallucination et c'est dans la seconde clause que l'on ajoute que cette représentation doit être vraie. De fait, le vocabulaire avec lequel Chrysippe a exposé la distinction entre représentation et hallucination n'est pas le même que celui avec lequel Zénon a décrit la représentation perceptive. Zénon, dans sa définition, parle d'une représentation qui «provient de ce qui existe», tandis que Chrysippe, dans la distinction entre hallucination et représentation, parle d'un «objet de représentation » ou phantaston qui «produit la représentation $\gg(\pi \circ 10 \tilde{\nu} \nu \tau \grave{\nu} \nu \alpha \nu \tau \alpha \sigma i \alpha \nu)$, ce qui implique clairement un sens causal : une représentation se distingue d'une hallucination en ce qu'elle est causée par un objet extérieur, et non par un simple mouvement à vide de l'âme ${ }^{45}$. La distinction entre représentation et hallucination proposée par Chrysippe est une explication en termes causaux, visant la manière dont la représentation est produite, puisqu'elle se réfère à la « production » de la représentation par son objet. Or une telle préoccupation semble absente de la définition de Zénon et de son commentaire rapporté par Sextus, qui s'intéressent seulement à l'existence ou non d'un objet de la représentation, et non à la question de savoir s'il a effectivement produit cette représentation. On peut douter, comme l'a souligné D. Sedley, que la préposition apo 
(«qui provient de... ), dans la définition de la représentation compréhensive, ait un sens causal, contrairement à ce que l'on admet souvent ${ }^{46}$. Peutêtre ne faut-il pas pour autant y voir un sens «représentatif» de apo comme le soutient Sedley ${ }^{47}$, car qu'un tel sens existe ne paraît pas indubitablement convaincant ${ }^{48}$. Et Sextus témoigne qu'à une certaine époque de l'histoire du stoïcisme, une définition redondante et circulaire de «ce qui existe » a été avancée, puisque les stoïciens ont soutenu que «ce qui existe est ce qui suscite une représentation compréhensive $\gg{ }^{49}$. À ce point, comme le souligne Sextus, la définition devient parfaitement circulaire, mais elle confirme qu'à partir du moment où Chrysippe a distingué la représentation de l'hallucination en disant que la première était produite par un objet extérieur, alors que la seconde n'était produite que par un mouvement à vide de l'âme, la clause initiale de la représentation compréhensive a été interprétée dans le sens de la distinction entre la représentation et l'hallucination comme posant que la première condition est que la représentation ne soit pas une hallucination. Comme cette distinction et la connotation causale qu'elle contient n'étaient pas déjà articulées par Zénon (ce qui ne l'empêcha pas de devoir affronter l'argument du rêve, puisque c'est précisément l'un

46. Sedley 2005, p. 76-77.

47. Voir Sedley 2005, p. 82-86. Sedley interprète notamment le passage de Sextus, $M$. VII, 245, où Sextus discute le cas du rêveur qui « forge à partir de Dion qui est vivant (ànò $\Delta i \omega v \circ \zeta \zeta \bar{\omega} v \tau \circ \varsigma)$ une attraction fausse et vide comme à partir de quelqu'un qui est à côté de lui ». Selon lui (p. 83), « dans ce passage, la préposition "à partir de" dans "à partir de Dion qui est vivant" ne signifie pas que l'impression est causée par le Dion vivant, mais qu'elle représente le Dion vivant, ou, peut-être plus explicitement, qu'elle représente le Dion vivant comme vivant $\gg$.

48. Il est certain que l'apparition de Dion dans les rêves n'est pas causée par Dion, puisqu'il y a précisément une attraction à vide sans que Dion la produise directement : la cause n'est pas Dion, mais un mouvement intérieur de l'âme. Mais que cet exemple et sa description puissent être attribués à Zénon parait pour le moins douteux. En outre, si l'attraction à vide se produit, c'est bien à partir d'une représentation de Dion qui s'est imprimée antérieurement dans l'âme, qui y réapparaît et qui y est remise en mouvement sans stimulation extérieure. Il faut donc distinguer la cause immédiate de l'hallucination (le mouvement à vide) de son origine : la préposition à $\operatorname{có}$ n'exprime pas la cause de la représentation, mais elle a peut-être moins un sens représentatif que celui de l'expression d'une provenance. Il ne faut peut-être pas trop surcharger la forme comprimée de l'argument de Sextus : on peut très bien lire l'argument comme disant que c'est à partir d'un Dion qui est bel et bien un être vivant (et qui n'est pas un être imaginaire) et qui a laissé son empreinte dans l'âme qu'il se produit dans l'âme du rêveur un mouvement à vide qui donne l'impression de voir Dion: autrement dit l'image de Dion vivant. Et dans ce cas, il n'y a pas d'usage représentatif de apo: pour que celui-ci soit établi, il faudrait qu'un texte dise d'un objet imaginaire (comme une Furie) ou d'un objet non sensible comme les dieux que nous avons une phantasia apo touton, mais, de fait, dans le cas d'une Furie, Sextus s'exprime sur le mode du « comme si... » ( $\dot{\omega} s$ ảmò ’Epivios, $M$. VII, 245).

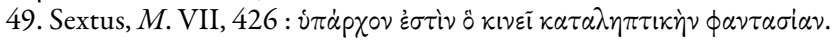


de ceux qu'Arcésilas paraît avoir déployés contre lui), il est probable que cette clause avait un sens plus large chez Zénon, et qu'elle visait à la fois le fait qu'une représentation perceptive devait avoir un objet existant, et qu'elle devait se représenter cet objet tel qu'il existe - sans aller toutefois jusqu'à affirmer que cette conformité allait dans les détails, ce qui est réservé à la seconde clause.

\section{B. La représentation perceptive reproduit fidèlement son objet}

La deuxième caractéristique de la représentation perceptive est qu'elle

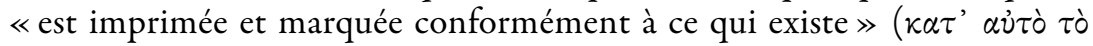

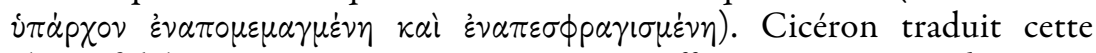
clause fidèlement en écrivant: «impressum effictumque ex eo unde esset ». Sextus l'explique ainsi :

La seconde propriété, c'est non seulement de provenir d'un objet existant, mais de lui être en outre conforme. Car il y a aussi des représentations qui proviennent d'un objet existant mais ne lui ressemblent pas, comme on l'a montré un peu auparavant à propos de la folie d'Oreste. [...] Mais encore c'est une représentation imprimée et marquée, afin que toutes les particularités des représentables soient reproduites avec art. De même que les graveurs s'appliquent à toutes les parties de ce dont ils font la finition, et de même que les sceaux portés aux anneaux impriment toujours tous leurs caractères dans la cire, de même ceux qui perçoivent les objets doivent parvenir à en saisir toutes les particularités. (M. VII, 249-251.)

Dans l'explication de cette seconde partie de la définition donnée par Sextus, il y aurait donc deux points: que la représentation soit vraie, et qu'elle soit en tous points fidèle à son objet. Comme cela vient d'être indiqué, il n'est pas certain que cette décomposition de la clause en deux éléments soit conforme aux intentions initiales de Zénon.

Le premier point expliqué par Sextus est qu'il ne suffit pas qu'il y ait un objet de la représentation, mais qu'il faut aussi que la représentation lui soit conforme. Le développement de Sextus renvoie ici à la folie d'Oreste. Dans le contexte de l'analyse de la représentation et de l'hallucination, Chrysippe citait des vers de l'Oreste d'Euripide ${ }^{50}$. Or, dans la pièce d'Euripide, la folie d'Oreste connaît deux étapes différentes : lorsque Oreste reconnaît Électre et s'imagine voir à côté d'elle des Érinyes, il est victime d'une hallucination, puisqu'il voit des Érinyes là où il n'y a personne ${ }^{51}$; mais, lorsque Électre lui saisit le bras pour calmer sa panique, il la voit sous l'apparence d'une Érinye $^{52}$. Il s'agit alors de ce que Sextus appelle une représentation à la fois

50. Voir sur ce point [Plut.], Plac. IV, 12, 900 F-901 A (SVF II 54).

51. Euripide, Or. 255-267 : ce sont les vers cités par Plac. IV, 12, 900 F-901 A.

52. Ce sont les vers auxquels se réfère Sextus, $M$. VII, 244-245. 
vraie et fausse: elle provient d'un être existant mais ne lui est pas conforme $e^{53}$. Cette conformité à son objet ne suffit pourtant pas à définir la représentation perceptive. Car cela n'en fait qu'une représentation vraie.

Le second point consiste dans l'exactitude et la précision de la représentation. Sextus dit que «toutes les particularités des représentables sont re-

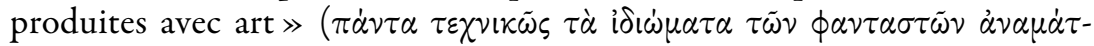
$\left.\tau \eta \tau \alpha_{l}\right)$. Sextus s'exprime plusieurs fois de la même manière, en disant que ce sont toutes les propriétés de l'objet qui sont reproduites dans la représentation (M. VII, 248, 250, 251). Mais Cicéron nuance cette affirmation en disant qu'il s'agit en fait de toutes les propriétés «qui peuvent tomber dans la perception » (quod cadere in eam potest) et non de «toutes celles qui sont dans la chose $\gg$ (omnia quae essent in re: Ac. Post. I, 42). Cicéron ne s'explique par sur le sens qu'il faut donner à cette restriction, mais l'hypothèse formulée par Frede 1987, p. 161, est crédible: une représentation sensible ne saisit pas toutes les propriétés d'un objet, mais seulement celles qui relèvent du sens par l'intermédiaire duquel elle nous parvient, ainsi les propriétés visibles pour la vision, ou les propriétés sonores pour l'audition ${ }^{54}$. Peut-être cela veut-il dire aussi qu'il y a, dans les cas de représentations sen-

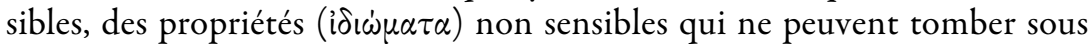
la perception, dans la mesure où elles ne sont pas immédiatement données dans la représentation perceptive, au moins dans la représentation perceptive sensorielle (par exemple le fait qu'il y a des pores est connu uniquement par déduction ${ }^{55}$ ) : de fait, selon la classification de Diogène Laërce, VII, 5253 , seuls les sensibles sont conçus par une «rencontre» ou «chute» ( $\kappa \alpha \tau \grave{\alpha} \pi \varepsilon p i \pi \tau \omega \sigma \nu$ ) dans l'âme.

Quant à la clause selon laquelle la représentation reproduit les propriétés de son objet «avec art $\gg(\tau \varepsilon \chi \nu$ $\leftarrow \tilde{\omega} \varsigma)$, elle doit être rapprochée d'un argument stoïcien rapporté par Cicéron, celui de l'éducation des sens par les arts. Il s'agit en effet d'un argument par lequel les stoïciens cherchaient à prouver la possibilité de représentations perceptives : grâce à l'art (ars) et à l'exercice (exercitatio), les yeux des peintres voient des choses que nous ne voyons pas, et les oreilles des musiciens peuvent reconnaître un interprète

53. Sextus, $M$. VII, 245. Sur tout ceci, voir Gourinat 1996, p. 40-42.

54. Sur ce point, voir Calcidius, In Tim. 220 (SVF II 879).

55. Sextus, $P$. II, 142. Il n'est pas impossible qu'une telle extension de la notion de perception ou de compréhension à des représentations non sensorielles qui sont conclues d'un raisonnement soit postérieure à Zénon. On comprendrait alors que Zénon ait considéré que ces propriétés non sensibles ne peuvent pas « tomber dans la perception ». Mais on le comprendrait même si Zénon avait déjà admis l'existence de représentations perceptives non sensorielles: même dans ce cas, il fallait bien admettre une différence entre les deux et reconnaître qu'il y a des propriétés qui ne peuvent pas tomber dans une représentation perceptive sensorielle. 
au premier son de la flûte (Ac. Pr. II, 20). Sextus fait une comparaison avec le travail de précision des graveurs de sceaux, qui renvoie évidemment à la comparaison de la représentation à une impression. Mais, alors que sa comparaison n'est qu'une comparaison, Cicéron donne un véritable exemple de ce qu'est le caractère «artistique » de la représentation perceptive.

\section{C. la représentation perceptive ne peut pas être confondue avec une représentation fausse}

La troisième propriété de la représentation perceptive, c'est qu'elle est

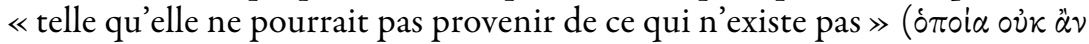

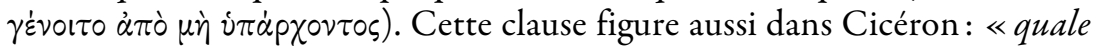
esse non posset ex eo unde non esset $\gg$. Selon Sextus, $M$. VII, 252, elle a été rendue nécessaire par les critiques des académiciens:

Quant à «telle qu'elle ne pourrait pas provenir de ce qui n'existe pas », ils l'ont ajouté parce que les académiciens n'ont pas admis comme les stoïciens qu'il serait impossible d'en trouver une qui soit totalement indiscernable $(\kappa \alpha \tau \dot{\alpha} \pi \dot{\alpha} \nu \tau \alpha \dot{\alpha} \pi \alpha \rho \dot{\alpha} \lambda \lambda \alpha \kappa \tau \dot{\partial} \nu \tau \iota v \alpha)$.

La même information se retrouve chez Cicéron, Ac. Pr. II, 77, qui précise que c'est une addition de Zénon lui-même, en réponse aux attaques d'Arcésilas ${ }^{56}$. L'argument académicien dit «des indiscernables » prenait deux formes, l'une selon laquelle il existe des hallucinations qui sont indiscernables des représentations vraies (Sextus, M. VII, 402-408), et l'autre

56. Selon Striker 1997, p. 265, l'anecdote est dans son ensemble « trop belle pour être vraie ». Déjà Long-Sedley 1987, II, p. 245, notent les qualifications de Cicéron : fortasse (« peut-être »), credo (« je crois »). Voir néanmoins Ioppolo 1997, p. 63 n. 3, pour qui il s'agit seulement de doutes quant à la littéralité des termes exacts employés par Zénon, mais l'historicité ne peut guère être mise en doute. À mon sens, il s'agit plutôt des termes d'un débat philosophique qui n'a jamais donné lieu à une discussion de visu entre les deux philosophes. La version donnée par Numénius semble indiquer qu'il n'y eut jamais d'affrontement direct, et même que Zénon ne répondit qu'indirectement à Arcésilas : « Arcésilas, voyant que Zénon était son rival professionnel et était en position de l'emporter, terrassa les discours qu'il prononçait sans aucun scrupule. [...] ce dogme que Zénon avait été le premier à inventer, la représentation compréhensive, comme il voyait que le dogme lui-même et le nom avaient une très bonne réputation à Athènes, il se servait de tous les moyens contre ce dogme. Quant à lui, qui était dans la position la plus faible et qui ne pouvait subir de tort s'il restait tranquille, il se tint à l'écart d'Arcésilas, alors qu'il aurait peut-être eu bien des choses à dire, mais il ne le voulut pas, ou peut-être fut-ce plutôt pour une autre raison et il livra combat contre l'ombre de Platon, qui n'était plus parmi les vivants. » (Eusèbe, P. E. XIV, 6, 12-13 = Numénius, fr. 25 Des Places $=S V F$ I 12.) Même si la version de Numénius tend à laisser penser que l'affrontement fut moins direct que ne le présente Cicéron, et que c'est sans doute pour cette raison qu'il présente le dialogue entre Zénon et Arcésilas comme une saynète fictive, le témoignage de Sextus, $M$. VII, 252, qui fait de l'addition de la clause une réponse aux pressions des académiciens, confirme sur ce point la version de Cicéron ( $c f$. Ioppolo 1997, p. 64 n. 6 ; voir Frede 1987, p. 163 ; Frede 1999, p. 299). 
selon laquelle il existe des objets indiscernables les uns des autres, par exemple les œufs (Sextus, M. VII, 409-410), mais aussi les jumeaux et les objets produits en série, comme les statues et les cachets imprimés dans la cire (Cicéron, $A c$. Pr. II, 85-86). Il en résulte qu'il ne peut pas y avoir de représentation perceptive, parce que l'on peut confondre une hallucination avec une vraie représentation et parce que la représentation de certains objets identiques ne permet pas d'y retrouver les propriétés distinctives de cet objet, et de le distinguer d'un autre: la première forme d'indiscernabilité remet en cause la première clause de la définition ( $c f$. Sextus, $M$. VII, 408), tandis que la seconde remet en cause la deuxième ( $c f$. Sextus, $M$. VII, 409).

Il semble que, sous cette forme double, l'argument des indiscernables puisse être attribué à Carnéade, dont Sextus, avant d'exposer les deux arguments de l'indiscernabilité, dit qu'il n'admettait pas la clause selon laquelle la représentation perceptive est « telle qu'elle ne pourrait pas provenir de ce qui n'existe pas » (M. VII, 402). En revanche, quand il explique la troisième clause, dans le texte cité ci-dessus (M. VII, 252), Sextus limite le problème des indiscernables à la représentation, et ne mentionne pas les objets indiscernables. Cela semble confirmé par Cicéron, selon qui Zénon n'aurait introduit la troisième clause que pour répondre à l'argument des représentations indiscernables formulé par Arcésilas: Arcésilas aurait demandé ce qui se passerait si une représentation fausse était de même qualité qu'une représentation vraie, et Zénon aurait donc ajouté que, pour qu'une représentation soit compréhensible, il fallait qu'elle ne puisse être confondue avec une représentation «provenant de ce qui n'est pas» $\left(A c\right.$. Pr. II, $\left.77^{57}\right)$. Cependant, tout en soulignant que la représentation perceptive a un caractère distinctif qui la différencie des autres représentations, Sextus mentionne aussi que « celui qui a la représentation compréhensive discerne de façon technique la différence entre les choses qui la sous-tend $»^{58}$. Or le discernement «technique » ne renvoie pas à la première clause de la définition, mais à la seconde, de sorte que, selon cette explication, la troisième clause, en jouant sur les deux formes de discernabilité, joue un double rôle car elle renvoie bien aux deux premières clauses de la définition. Dans la forme de la polémique attribuable à Carnéade, on constate à la fois une distinction assez nette entre les deux arguments et une tendance à affirmer que l'indiscernabilité des objets conduit à l'impossibilité de distinguer entre une représentation compréhensive et une représentation fausse et non

57. Selon Sedley 2005, p. 88-89, Zénon serait alors passé, sous la pression d'Arcésilas, d'un sens causal de apo dans la définition de la représentation perceptive à un sens représentatif.

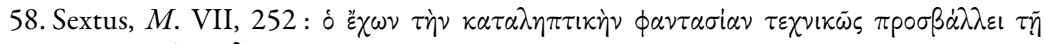

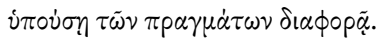


compréhensive ( $M$. VII, 409). Même si Cicéron ne mentionne quant à lui l'argument des indiscernables qu'à propos des objets, il tire du problème des indiscernables (par exemple des jumeaux) l'affirmation qu'il est impossible de distinguer une représentation vraie d'une représentation fausse.

\section{La représentation perceptive est un critère de vérité s'il n'y a pas d'obstacle}

Selon Sextus, un quatrième point a été rajouté ultérieurement aux éléments de cette définition par des «stoïciens plus récents ${ }^{59}$. Selon ces stoïciens, la représentation perceptive serait un critère de la vérité «si elle ne rencontre pas d'obstacle $(\varepsilon ้ \nu \sigma \tau \eta \mu \alpha) \gg(M$. VII, 254). Cette clause ne fait pas partie de la définition de la représentation perceptive, mais constitue une précision ajoutée à celle-ci. Cette clause était illustrée par deux exemples, celui d'Admète voyant revenir sa femme Alceste des Enfers, et celui de Ménélas retrouvant Hélène à la cour du roi Protée.

À l'issue des dix années de la guerre de Troie, Ménélas croit ramener Hélène chez lui mais il ignore qu'en réalité elle n'a jamais été enlevée par Pâris : Pâris n'a enlevé qu'un fantôme qui ressemble à Hélène. Celle-ci est depuis dix ans en Égypte, à la cour de Protée. Lorsque Ménélas rencontre la véritable Hélène, il n'en croit pas ses yeux. Autrement dit, il ne donne pas son assentiment à la représentation perceptive qu'il a d'Hélène. « Ménélas réfléchissait qu'il avait laissé Hélène sous bonne garde à bord de son vaisseau et qu'il n'était pas incroyable que celle qu'il venait de découvrir à Pharos ne fût pas Hélène mais un démon imaginaire $(\phi \dot{\alpha} \nu \tau \alpha \sigma \mu \alpha \delta \dot{\varepsilon} \tau \iota \kappa \alpha \grave{l}$

59. Sextus, $M$. VII, 253, se réfère seulement à des «stoïciens plus récents ». Comme l'exemple de Ménélas était également utilisé par Carnéade pour illustrer ce qu'il appelait une « représentation non empêchée $(\dot{\alpha} \pi \varepsilon p i \sigma \pi \alpha \sigma \tau o \varsigma \phi \alpha \nu \tau \alpha \sigma i \alpha) »(M$. VII, 180), on pense généralement que cet exemple a été repris à Carnéade par les «stoïciens plus récents 》 (voir par exemple Striker 1990, p. 152, n. 14). Il en résulte que les stoïciens « plus récents » pourraient en fait être Antipater, comme le remarque Sandbach 1971, p. 14. Antipater, contemporain de Carnéade, est en effet connu pour avoir passé beaucoup de temps à réfuter les académiciens (Cicéron, Ac. Pr. II, 17), et plus précisément pour avoir écrit de nombreux livres contre Carnéade (Plutarque, Garrul. 23, $514 \mathrm{D}=S V F$ III Ant. 5 ; cf. Cicéron, Ac. Pr. II, 28). On remarquera toutefois que Sextus attribue deux exemples aux stoïciens et un seul à Carnéade, ce qui semble indiquer que la version stö̈cienne est la plus complète. En outre, ces deux exemples sont empruntés à Euripide (l'histoire d'Admète est rapportée dans l'Alceste, celle de Ménélas est le thème de l'Hélène), ce qui est caractéristique de Chrysippe, comme on l'a vu ci-dessus, p. 57 et n. 38. Enfin, on sait que Chrysippe a beaucoup écrit contre les sens (Cicéron, Ac. Pr. II, 75), et qu'il a fourni des arguments à Carnéade (Ac. Pr. II, 87). Il n'est donc pas impossible que Carnéade ait repris l'exemple de Ménélas à Chrysippe (on voit mal pourquoi Antipater aurait ajouté un exemple négligé par Carnéade et cherché à renforcer les critiques de celui-ci). À mon sens, aucun argument n'est vraiment décisif, mais Antipater est malgré tout le protagoniste le plus vraisemblable de l'affaire, car la désignation de «stoïcien plus récent » paraît mieux s'appliquer à lui qu'à Chrysippe ( $c f$. Ioppolo 1986, p. 201 n. 28). 


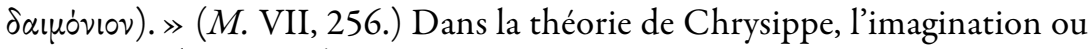
phantasme $(\phi \dot{\alpha} \nu \tau \alpha \sigma \mu \alpha)$ est « ce vers quoi nous sommes attirés dans l'attraction à vide de l'hallucination ${ }^{60}$, de sorte que l'on retrouve à nouveau le vocabulaire chrysippéen de la distinction entre représentation et hallucination, sans que cette distinction soit faite. Quant à Admète, il avait beau se former une représentation perceptive d'Alceste, il ne voulait pas non plus reconnaitre sa femme qu'Hercule ramène des Enfers car il «faisait le raisonnement qu'Alceste était morte et qu'un mort ne revient jamais, mais que certains démons font quelquefois des apparitions ». Des obstacles comme les croyances ou les raisonnements empêchent donc que Ménélas et Admète ne reconnaissent leurs femmes. Ils ont donc bien une représentation perceptive de la réalité, mais cette représentation perd son caractère irrésistible et n'agit pas comme critère de la vérité.

La différence entre l'analyse de l'exemple de Ménélas par Carnéade et par les «stoïciens récents» est significative de la polémique engagée entre stoïciens et académiciens. Carnéade considère que «le jugement de la vérité est fait d'après la concurrence des représentations », et que, dans le cas de Ménélas, il ne «fait pas confiance à la représentation vraie» d'Hélène parce qu'il en est «détourné » par une autre représentation, à savoir celle qu'il a laissé Hélène sur le navire: c'est pourquoi le critère est une représentation à laquelle aucune autre ne fait obstacle ( $\dot{\alpha} \pi \varepsilon p i \sigma \pi \alpha \sigma \tau \varsigma \varsigma)$, contrairement à celle-ci (Sextus, M. VII, 179-181). Les stoïciens récents considèrent que Ménélas a d'Hélène une représentation «qui provient de ce qui est, qui est imprimée et marquée conformément à ce qui est », c'està-dire une représentation compréhensive, mais qu'elle a rencontré un «empêchement 》 (है $\nu \sigma \tau \eta \mu \alpha)$, et cet empêchement consiste dans les raisonnements que fait Ménélas et dans ce qu'il «voit » qu'il n'est pas « incroyable $(\dot{\alpha} \pi i \theta \alpha v \circ v) \gg$ qu'il ait affaire à une hallucination (Sextus, M. VII, 255-257). Dans les deux cas, on a donc affaire à une représentation objectivement vraie, que les stoïciens considèrent comme perceptive, mais qui rencontre un obstacle. Dans le cas des stoïciens, la réaction de Ménélas est exactement inverse de celle que les académiciens dénoncent habituellement comme prouvant l'inexistence de la représentation perceptive: il n'y a pas une hallucination qui est confondue avec une représentation, mais une représentation perceptive qui est tenue pour une hallucination. Striker 1990, p. 152-153, n. 14, pense qu'il y a de la part des stoïciens une «grossière erreur » parce qu'en fait Ménélas n’a pas une vraie représentation d'Hélène, mais que sa «pensée » est bien plutôt «quelque chose comme

60. [Plutarque], Plac. IV, 12, 900 D. Cf. Diogène Laërce, VII, 50, où le phantasme est décrit plus limitativement comme « une vision de la pensée qui se produit dans les rêves »

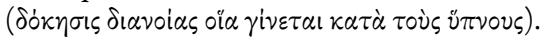


"ce doit être un fantôme" ou "ce ne peut être Hélène" ». Mais ce que soutiennent les stoïciens, c'est précisément que Ménélas a une représentation conforme d'Hélène, mais qu'il « raisonne » et « réfléchit » différemment, et que, pour cette raison il ne donne pas son assentiment à la représentation initiale - autrement dit, ils font une distinction entre la représentation que reçoit Ménélas, et les pensées avec lesquelles il l'interprète. Il y a donc d'un côté une impression sensible correcte, et d'autre part un raisonnement et une réflexion erronés. La proposition «crédible » que Ménélas formule à propos de la vision de la véritable Hélène, qui est qu'il ne s'agit que d'un fantôme, n'est pas le contenu brut de sa représentation : la représentation que Ménélas a d'Hélène est vraie, mais il la croit fausse et donne son assentiment à une autre représentation. Contrairement à Carnéade, qui limite le cas de Ménélas à une représentation qui en bloque une autre, et empêche la croyance, sans qu'intervienne nullement dans ce contexte l'enjeu de l'existence de la représentation compréhensive, les stoïciens font apparaître une erreur de raisonnement et une représentation crédible: dans la version de Carnéade, il y a seulement deux représentations, celle de l'Hélène sur le bateau, et celle de l'Hélène à Pharos; dans la version stoïcienne, il y a en plus la représentation «crédible» (mais fausse) que l'Hélène à Pharos n'est qu'un fantôme. Bien que ce ne soit pas dit par Sextus, il est clair que l'erreur initiale de Ménélas, qui a donné antérieurement son assentiment à une représentation non compréhensive - lorsqu'il a cru que l'Hélène de Troie était son épouse -, est ce qui l'empêche désormais de donner son assentiment à la représentation compréhensive qui se présente à lui. De ce fait, la représentation compréhensive ne joue pas son rôle de critère de la vérité.

\section{Arguments pour et contre la perception et la représentation perceptive}

Le principe de la polémique entre stoïciens et académiciens a manifestement été fixé par Arcésilas, ainsi qu'en témoigne Cicéron, Ac. Pr. II, 77-78:

Zénon a vu avec beaucoup de perspicacité qu'il n'y aurait aucune représentation qui puisse être perçue, si celle qui provient de ce qui est pouvait être du même mode que celle qui provient de ce qui n'est pas. Arcésilas admit à juste titre la définition complétée, et reconnut que le faux ne peut être perçu, et que le vrai ne peut pas l'être non plus si le vrai est tel que le faux. Il s'engagea donc dans ces discussions destinées à montrer qu'il n'y a pas de représentation qui vienne du vrai qui ne puisse pas être semblable à celle qui vient du faux. C'est là l'unique point de débat qui a perduré jusqu'à nos jours.

Or nous connaissons deux aspects de la critique académicienne. L'un est le bref résumé des arguments d'Arcésilas contre la perception rapporté par 
Sextus. L'autre est le vaste ensemble d'arguments détaillé par Cicéron dans le Lucullus et Sextus dans le livre VII de l'Adversus Mathematicos. On notera certes que la version que nous donne Cicéron est celle d'Antiochus, qui n'est pas un stoïcien, mais un académicien, et il s'agit donc d'une version filtrée et modifiée qu'il serait imprudent de prendre pour argent comptant $^{61}$. Mais il n'en reste pas moins vrai que dans ces «développements longs et variés » $(A c$. Pr. II, 41), on ne trouve au fond que l'« unique point de débat » résumé par Cicéron. Cependant, les arguments d'Arcésilas lui-même portent autant sur la notion même de perception que sur l'existence de représentations perceptives, tandis que les arguments postérieurs mettent l'accent sur l'inexistence des représentations perceptives et généralisent ce scepticisme par une thèse selon laquelle il est impossible de se fier aux sens qui nous ont une fois trompé - un principe tout aussi radical que contestable.

\section{A. Les arguments d'Arcésilas}

Sextus attribue trois arguments contre la perception à Arcésilas. Le premier vise le statut intermédiaire de la perception entre la science et l'opinion : Arcésilas remarque que la science et l'opinion s'opposent comme les sages et les incultes, mais que, comme les stoïciens ne reconnaissent pas d'intermédiaire entre ces deux catégories d'individus, alors la perception ne peut pas exister, car elle ne pourrait appartenir qu'à l'une de ces deux catégories et n'a pas d'existence possible (Sextus, $M$. VII, 153). L'argument est assez faible, puisque les stö̈ciens considèrent précisément que la perception est commune aux sages et aux fous - mais peut-être ce point at-il été avancé en réponse à l'argument d'Arcésilas. Les deux autres arguments attaquent la possibilité de la perception en attaquant la notion de perception comme assentiment à la représentation perceptive. Le premier est que l'assentiment porte sur les propositions et non sur les représentations, et le second qu'il n'y a « aucune représentation vraie qui ne serait pas susceptible de devenir une représentation fausse $\gg$, autrement dit qu'il n'y a pas de représentation perceptive ${ }^{62}$. Ce troisième argument est celui dont Cicéron dit qu'il a perduré tout au long de l'histoire de la polémique entre stoïciens et académiciens. Le second argument n'est attesté que de la part d'Arcésilas.

Le second argument consiste en une déconstruction de la notion de perception, pour montrer que, définie comme assentiment à la représenta-

61. C'est ce que souligne Striker 1997, p. 258. Brittain 2012 tend néanmoins à montrer que la position épistémologique d'Antiochus est presque entièrement conforme à celle des stoïciens.

62. Sextus, $M$. VII, 154 (Arcesilas, F. 11 Mette 1984), cité plus haut p. 53-54. 
tion perceptive, elle est inconsistante et contradictoire. Arcésilas objecte que l'assentiment porte sur des propositions et non sur des représentations, et cherche à montrer par là que la notion de perception, parce qu'elle suppose un assentiment à un genre particulier de représentation, n'est pas consistante avec la notion d'assentiment. Selon Couissin 1929, Arcésilas procédait par des réfutations ad hominem, c'est-à-dire en retournant contre son adversaire stoïcien certaines de ses propres thèses. C'est ainsi, que, dans le cas de l'argument sur l'objet de l'assentiment, Arcésilas semble faire appel à sa propre théorie, selon laquelle l'assentiment porterait sur une proposition, mais il se pourrait bien qu'il ait tiré cette formule d'un dogme formulé par Zénon ${ }^{63}$. Couissin renvoie au passage de Stobée, Ecl. II, 9, p. 88, 4 (SVF III 171), cité plus haut (p.53) et au fait que les stoïciens considèrent en général que c'est la proposition qui est vraie ou fausse (Diogène Laërce, VII, 65). Mais l'usage logique du terme «proposition » $(\dot{\alpha} \xi i \omega \mu \alpha)$ n'est pas attesté clairement pour un stoïcien avant Chrysippe, de sorte que le passage de Sextus sur l'objection d'Arcésilas est le seul qui puisse donner l'indication d'un usage du terme par Zénon lui-même. Quant au terme «assentiment», il n’est pas attesté avant Zénon et Arcésilas, de sorte qu'il est difficile de déterminer un usage antérieur du terme auquel Arcésilas pourrait se référer: mais on peut toutefois remarquer que l'usage du verbe correspondant, $\sigma \nu \gamma \kappa \alpha \tau \alpha \tau i \theta \varepsilon \nu \alpha$, signifie « donner son consentement », et ne vise pas des représentations ou des sensations, mais des opinions exprimées par la parole ${ }^{64}$. De toute évidence, l'emploi du terme « assentiment » appliqué à des représentations est, par rapport à l'usage du verbe «donner son consentement », un usage excentrique, sinon idiosyncrasique, et il est donc assez vraisemblable qu'Arcésilas reproche à Zénon de faire un usage déplacé du terme «assentiment » qui, comme il le dit, «ne s'applique pas à la représentation mais au langage » (Sextus, M. VII, 154) et, dans ce cas, la parenthèse selon laquelle les assentiments portent sur des propositions pourrait bien être une simple glose de Sextus. La thèse d'un assentiment aux représentations, probablement instaurée par Zénon, serait quelque chose à quoi Arcésilas objecterait l'usage courant du verbe correspondant ${ }^{65}$.

63. Couissin 1929, p. 243 (Couissin 1983, p. 33).

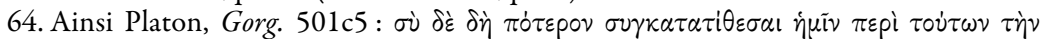

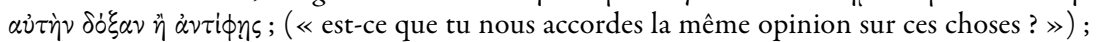

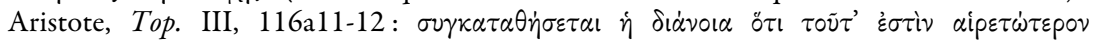
(«l'esprit reconnaît que c'est un meilleur choix »); Démosthène, Sur la couronne, 166

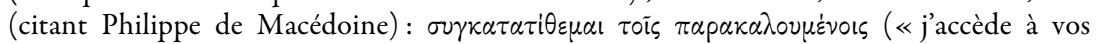
demandes $\gg$ ).

65. Schofield 1999, p. 328, voit dans l'objection d'Arcésilas la réutilisation de l'argument du Théétète contre l'idée que la vérité est accessible à la perception sensible. 
Il est donc possible que ce soit Chrysippe qui ait développé une version plus complexe de la doctrine de l'assentiment dans laquelle il aurait intégré l'objection d'Arcésilas en cherchant à articuler l'assentiment à la représentation et l'assentiment à la proposition. D'autre part, il semble qu'en liant la représentation et son contenu exprimable dans la définition de l'exprimable $^{66}$, Chrysippe a dû montrer que l'alternative entre l'assentiment à la représentation et l'assentiment à la proposition n'en est pas vraiment une. Ce faisant, il a dû neutraliser l'argument d'Arcésilas, comme semblent l'indiquer le peu d'importance qui lui est accordé par les auteurs ultérieurs (quelques lignes chez Sextus, rien dans ce qui nous reste de l'argumentation de Cicéron) et le fait que ce point du débat n'ait pas perduré.

La tactique d'Arcésilas consiste manifestement à prendre la définition de Zénon et à en réfuter chaque élément. Zénon dit que la perception est un assentiment à une représentation perceptive: Arcésilas soutient donc, pour déconstruire la notion de perception, que l'assentiment ne porte pas sur les représentations et qu'il n'y a pas de représentation perceptive. Il nie séparément chaque élément de la définition, de sorte que même si l'un des deux éléments était rétabli, la réfutation de l'autre élément resterait valide. Toujours selon Sextus, M. VII, 154, l'argumentation d'Arcésilas faisait sur le deuxième point appel à des «exemples nombreux et variés » pour montrer qu'il n'y avait pas de représentation perceptive. D'après l'explication de Sextus, $M$. VII, 252, selon laquelle, pour les académiciens, il est « impossible de trouver une représentation indiscernable à tous égards », et puisque c'est à cause de cette objection que Zénon avait formulé la dernière partie de sa définition, les exemples nombreux et variés d'Arcésilas devaient contenir déjà une partie au moins des exemples d'hallucinations indiscernables des représentations vraies, exemples qui semblent fournir la partie la plus ancienne de la polémique, dans la mesure où elle est peut-être à l'origine de la distinction entre représentation et hallucination opérée ultérieurement par Chrysippe.

\section{B. Objections académiciennes et défense stoïcienne: la polémique détaillée}

Comme l'explique Cicéron dans les $₫$ 40-41 du livre II du Lucullus, l'essentiel des arguments détaillés des académiciens consiste à admettre une distinction entre les représentations vraies et les représentations fausses, et à soutenir qu'il est impossible de distinguer les unes des autres. Tous ces arguments reprennent donc le principe du second argument d'Arcésilas. Cela signifie que, dans la définition de la représentation perceptive par Zénon, les académiciens acceptaient la deuxième clause mais qu'ils reje- 
taient la troisième. Ils ne niaient pas qu'il y eût des représentations vraies qui reproduisent fidèlement leur objet, ils niaient qu'il fût possible de reconnaître une telle représentation et qu'il fût possible de la distinguer d'une représentation fausse et illusoire.

Il est difficile de savoir à quels stoïciens et à quels académiciens doit être attribuée la mise en forme des arguments pour et contre que l'on trouve dans les Académiques et dans Sextus. Une partie au moins est nécessairement une version tardive, révisée et filtrée par Antiochus, et n'est pas authentiquement stoïcienne, ce que Cicéron ne prétend pas ${ }^{67}$. Cicéron se réfère partiellement à Clitomaque, un disciple de Carnéade qui avait rapporté ses arguments (Ac. Pr. II, 98). Il semble donc que la forme canonique des arguments était au moins partiellement établie du temps de Carnéade, mais on ne peut guère savoir si oui ou non elle l'était déjà avant. Quant à Carnéade lui-même, puisqu'il n'a rien écrit, le filtre imposé par le compterendu de Clitomaque introduit une incertitude supplémentaire ${ }^{68}$. En outre, on sait qu'Antipater a beaucoup écrit contre Carnéade: les objections d'Antipater ont dû cristalliser une certaine version de la tradition ${ }^{69}$. Enfin, on sait que Chrysippe a beaucoup écrit contre les sens (Cicéron, Ac. Pr. II, 75), et qu'il a de ce fait fourni des arguments à Carnéade (Ac. $\operatorname{Pr}$. II, 87). Il est donc possible que les arguments systématiquement développés par les académiciens l'aient été par Carnéade, qui aurait ainsi retourné contre les stoïciens des analyses empruntées à Chrysippe.

On a affaire en tout cas à une longue série d'arguments pour et contre, qui se répondent souvent très précisément, sauf lorsque les académiciens sont engagés dans une polémique contre les épicuriens. Certaines analyses d'Épicure ou des épicuriens sont en effet communes aux stoïciens et aux épicuriens, tandis que les stoïciens refusent parfois de se laisser inquiéter par d'autres analyses épicuriennes, utilisées par les académiciens contre eux.

Cette longue série d'arguments peut être divisée en quatre grandes catégories. Le tableau en annexe (p.86) fournit une représentation synthétique des arguments stoïciens et académiciens, disposés en colonnes parallèles. Ce sont les arguments et les exemples que l'on retrouve dans les Académiques et dans Sextus et, pour certains, dans le Contra Academicos d'Augustin. Quelques arguments proviennent d'autres sources.

La première catégorie d'arguments, celle des erreurs des sens, est une attaque contre la seconde clause de la définition de la représentation per-

67. Voir les remarques de Striker 1997, p. 258, déjà mentionnées ci-dessus n. 61.

68. En ce qui concerne la position philosophique de Carnéade, Cicéron avait à sa disposition deux autres versions, celle de Philon et celle de Métrodore (Ac. Pr. II, 78). Sur les différences entre ces versions, voir Schofield 1999, p. 334-338.

69. Voir n. 59 supra. 
ceptive, celle de la conformité des représentations sensorielles à l'objet qui les produit. Il faut y distinguer entre ce que les stoïciens appellent des représentations fausses, qui sont des erreurs normales des sens dues aux conditions de la représentation sensorielle ${ }^{70}$, et ce qu'ils appellent les représentations vraies et fausses, qui sont des erreurs dues au dérèglement des sens dans la folie (Sextus, M. VII, 244-245). Seules les secondes sont d'ailleurs répertoriées comme des objections académiciennes par Sextus.

Les représentations fausses sont des exemples qui apparaissaient aussi dans le contexte de la polémique entre épicuriens et académiciens: d'après Cicéron, c'est notamment le cas de celui du cou du pigeon et de la rame brisée. Lucrèce, II, 801, confirme l'usage par les épicuriens de l'exemple du cou du pigeon qui paraît multicolore. Cet exemple ne troublait pas les stoïciens, car il ne s'agissait pas d'une illusion qui induisait en erreur: «Je ne dis pas que tout ce qui m’apparaît est tel que cela m’apparaît. » (Cicéron, Ac. $P r$. II, 19.) La position académicienne consiste à faire remarquer qu'il m'apparaît des choses qui n'existent pas (II, 79), ce qui est supposé remettre en cause la fiabilité des sens. Mais les stoïciens admettent l'existence de telles représentations fausses (Sextus, $M$. VII, 244) et se contentent de faire remarquer qu'il faut précisément distinguer entre ces représentations fausses et les représentations vraies. Un autre argument, celui de la couleur de la mer, que l'on trouve en $A c$. Pr. II, 105, semble avoir exclusivement appartenu à la polémique académico-épicurienne ${ }^{71}$. L'argument de la taille du soleil est un argument utilisé par les académiciens contre les épicuriens: Cicéron rappelle en effet que les mathématiciens démontrent que le soleil est dix-huit fois plus grand que la terre alors qu'Épicure, pour maintenir la thèse de la vérité des sensations, pense qu'il peut même être plus petit qu'il n'y parait ${ }^{72}$. Les stoïciens n'avaient guère de raison de prendre parti dans cette querelle, mais ils l'ont fait, en y ajoutant comme Épicure l'exemple du feu des étoiles, qui paraît tout petit ${ }^{73}$. D'après Calcidius, les stoïciens

70. Leibniz remarque à juste titre que ces « fausses apparitions... viennent... de la nature de la vision même » (Théodicée, disc., 64).

71. On trouve en effet cet exemple développé par Lucrèce dans le De Natura Rerum, II, 766 sq. Il est possible que Cicéron, qui avait lu Lucrèce ( $Q . f r$., II, 9 (10), 3), et qui en avait peut-être même été l'éditeur (Jérôme, Chron. ad annum 94 a), l'ait trouvé dans le $D e$ Natura.

72. Cicéron, Ac. Pr. II 82. L'argument se trouve en effet chez Épicure, Lettre à Pythoclès, 91. C'est Épicure qui soutiendrait que « tout ce qui m’apparaît est tel que cela m'apparaît ».

73. Calcidius, in Tim. 237 (SVF II 863). C'est sous cette forme que Descartes reprendra l'argument : «Et parce que les étoiles ne lui faisaient guère plus sentir de lumière que des chandelles allumées, elle n'imaginait pas que chaque étoile fût plus grande que la flamme qui paraît au bout d'une chandelle qui brûle. » (Principes de la philosophie, I, 71 .) 
n'étaient pas en peine pour expliquer toutes ces illusions d'optique, ce qui permettait à la fois de comprendre que les choses ne nous apparaissent pas telles qu'elles sont et que l'on puisse néanmoins faire confiance à certaines représentations. C'est en effet le mécanisme de la vision par un cône visuel qui explique pour les stoïciens toutes ces déformations de la vision oculaire. La diminution de l'acuité visuelle avec la distance, et les déformations perspectives induites par le caractère conique de la vision font que les tours carrées paraissent rondes, que le portique paraît aller en diminuant et que le soleil et les étoiles paraissent tout petits ${ }^{74}$. Cette propriété de la vision ne doit pas pour autant entamer la confiance que l'on peut placer dans une vision perceptive qui répond à de bonnes conditions, et pour laquelle on fera par exemple varier la distance et la position (Cicéron, Ac. Pr. II, 19). Quant à l'argument des bâtiments immobiles qui paraissent bouger depuis un bateau (Cicéron, Ac. Pr. II, 81-82), il s'agit encore d'un argument que l'on retrouve chez Lucrèce, IV, 389-390. Augustin, Trin., XV, 12, 21, en a fait un argument des tours qui paraissent bouger depuis la terre ferme, en l'amalgamant avec l'illusion des tours carrées, qui était un des exemples favoris des épicuriens mais que l'on semble avoir retrouvé chez les stoïciens ${ }^{75}$. Il est donc remarquable que la plupart des éléments de la discussion relatifs aux représentations fausses ne sont pas spécifiques à la polémique académico-stö̈cienne mais sont des éléments empruntés aux analyses épicuriennes.

Comme cela a été indiqué plus haut (p.59 n. 40), les représentations vraies et fausses ne sont pas distinguées par Cicéron des hallucinations. L'un des arguments fondamentaux est certainement celui selon lequel les représentations perceptives sont des représentations saines, non pathologiques $^{76}$. Pour le reste, les arguments devaient être les mêmes que ceux qui réfutaient les arguments relatifs aux hallucinations.

La série des arguments sur les hallucinations indiscernables des représentations vraies constitue précisément la seconde catégorie d'arguments. C'est surtout elle qui a nécessité l'adjonction de la troisième clause, celle selon laquelle les représentations perceptives ne peuvent pas être identiques à celles qui proviennent de ce qui n'existe pas. Les arguments du rêve, du délire éthylique et de la folie ont en effet pour fonction de montrer que

74. Calcidius, in Tim. 237 (SVF II 863).

75. L'argument est attribué aux stoïciens par Calcidius, in Tim. 237 (SVF II 863) et aux épicuriens par Sextus, $M$. VIII, 208. Là encore, Descartes reprendra l'exemple : « des tours qui de loin m'avaient semblé rondes, me paraissent de près être carrées » (Méditations métaphysiques, VI, AT VII 76, IX 61).

76. Cicéron, $A$ c. Pr. II, 19; Sextus, $M$. VII, 247 ; 424. Sur l'importance de l'opposition entre le normal et le pathologique, voir Frede 1987, p. 157-158, 176-177. 
nous donnons notre assentiment à des hallucinations qui nous abusent parce qu'elles paraissent exactement identiques à des représentations perceptives ${ }^{77}$. La défense des stoïciens comportait trois points: le fait que les représentations du rêve manquent de la clarté de celle de la veille; le fait qu'au réveil ou après la crise de délire nous cessons de donner notre assentiment (il s'agit donc d'un assentiment faible et non d'un assentiment perceptif, ce qui prouve la différence entre les représentations) ; le fait que les fous et les mélancoliques ne donnent pas leur assentiment à leurs représentations vraies parce qu'elles ne sont pas perceptives, ce qui prouve que, malgré leur folie, ils savent que c'est à des représentations perceptives qu'il faut donner son assentiment (M. VII, 247). Le second point ressemble d'assez près à un argument d'Aristote dans la Métaphysique $(\Gamma, 5)$, selon qui

il est juste de s'étonner que l'on soit embarrassé de la difficulté de savoir [...] si sont vraies les choses qui apparaissent telles à ceux qui dorment ou à ceux qui sont éveillés. Car il est évident qu'ils ne le pensent pas réellement. En tout cas, il n'y a personne qui se mettra en route vers l'Odéon parce qu'il s'imagine une nuit se retrouver à Athènes, alors qu'il est en Libye. (1010b411.)

Les stoïciens, comme Aristote, pensent que les rêveurs ne croient pas vraiment à leurs rêves. Toutefois les stoïciens, si tant est qu'ils aient connu l'argument d'Aristote, y ajoutent la notion d'assentiment, qui n'apparaît pas chez Aristote. Pour Aristote, si le dormeur donnait son assentiment, il agirait en conséquence. Chez les stoïciens, l'argument de l'action n'est pas explicitement présent, mais ils soulignent qu'il n'y a pas dans le rêve d'assentiment stable (autrement dit, pas de perception), puisque c'est un assentiment instable, qui cesse au réveil, comme il cesse après une crise de folie ou d'éthylisme. La réponse stoïcienne à l'argument des hallucinations, c'est qu'il est clair, d'après le comportement de ceux qui sont objets d'hallucinations, qu'une hallucination n'entraîne pas la même réaction et le même assentiment qu'une représentation perceptive. En ce sens, tous ces cas apparaissent plutôt aux stö̈ciens comme des arguments qui prouvent qu'il est possible de discerner une représentation perceptive d'une hallucination.

Comme on l'a déjà vu plus haut, l'argument des objets indiscernables, quant à lui, attaque plutôt la clause de la conformité des représentations sensorielles à l'objet qui les produit. Cet argument suppose que nos sens ne

77. Il est troublant de noter que, dans le De trinitate d'Augustin, l'argument du délire éthylique a disparu et que ce sera également le cas dans les Méditations de Descartes. Descartes prétendait n'avoir lu le De trinitate qu'après la rédaction des Méditations, mais cette analogie entre les deux textes laisse planer le soupçon que ce n'était peut-être pas vrai. Il est toutefois possible qu'il ait été influencé par des auteurs dont l'exposé était inspiré par le $D e$ trinitate plutôt que par Cicéron. 
peuvent pas distinguer entre deux choses qui seraient «semblables quant à la forme mais différentes en ce qui concerne leur sujet $\gg^{78}$. Les deux cas cités à la fois par Cicéron et Sextus sont ceux des œufs et des jumeaux. L'argument stoïcien était identique dans les deux cas : il y a dans un individu quelconque quelque chose qui le distingue de son jumeau, et qui permet à sa mère de le reconnaître ${ }^{79}$, comme il y a quelque chose dans la représentation perceptive qui la distingue des images des rêves, des hallucinations des fous et des ivrognes. L'examen de tels cas et leur dispute semble remonter aux premières générations de stoïciens, notamment à Ariston et à Persaïos ${ }^{80}$, et c'est sans doute contre l'argument des indiscernables que Chrysippe a éla-

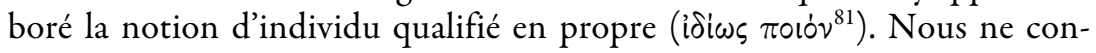
naissons pas la réponse que pouvaient donner les stoïciens à l'argument des objets produits en série. L'un d'entre eux, celui des cachets dans la cire, paraît pourtant viser directement la théorie stoïcienne, puisqu'il paraît renvoyer allusivement à la métaphore de l'impression. Il est remarquable enfin que l'argument des objets indiscernables n'apparaisse pas dans le doute augustinien ou cartésien, parce qu'il ne remet pas en cause l'existence du monde extérieur, mais seulement l'exactitude de notre représentation de celui-ci.

La quatrième catégorie d'arguments nie la possibilité de percevoir le sens de certaines propositions. Le contexte n'est plus celui des erreurs des sens ou des objets de représentations sensorielles, mais un contexte strictement dialectique. Cicéron donne comme exemples les sorites et l'argument du menteur, qui sont des sophismes à la réfutation desquels Chrysippe a consacré de longs développements, mais qui ne semblent pas avoir été connus de Zénon ou du moins pas discutés par lui ${ }^{82}$. Il les réfutait par une

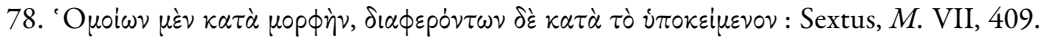
Cela semble être une affirmation de Carnéade.

79. Cicéron, Ac. Pr., II 56. Les éleveurs de poules de Délos étaient également capables de dire quelle poule avait pondu un œuf donné (Ac. Pr. II, 57).

80. Il est possible, comme le souligne Ioppolo 1997, p. 67-68, qu'Hermagoras d'Amphipolis, un disciple de Persaios de Citium, ait écrit un ouvrage sur l'examen des œufs, intitulé Le gaspillage ou Sur la sophistique, contre les académiciens (Suda s.v. Hermagoras). Arnim (SVF I 462) y voit deux ouvrages différents, mais A. M. Ioppolo souligne qu'il pourrait s'agir d'une attaque contre le temps passé par les académiciens à argumenter en faveur des indiscernables - notamment à propos de l'argument des œufs. Sur les jumeaux, voir l'anecdote racontée par Diogène Laërce, VII, 162 (SVF I 347), sur un piège tendu par Persaïos à Ariston, en lui envoyant deux frères jumeaux, l'un pour lui confier une somme d'argent et l'autre pour la récupérer (commentaire par Ioppolo 1997, p. 68-69).

81. Cf. Plutarque, Not. Com. 36, 1077 C-D, et Cicéron, Ac. Pr. II, 84-85. Voir Ioppolo 1997 , p. 65 n. 11.

82. Le sorite et le Menteur auraient été inventés par Eubulide de Milet selon Diogène Laërce, II, 108. Ils étaient discutés par Chrysippe (Sextus, P II, 253 ; Diogène Laërce, VII, 


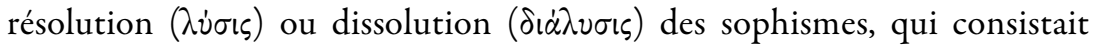
essentiellement à les démonter pour montrer le mécanisme de l'illusion sophistique.

À ces séries d'arguments opposant pied à pied des exemples divers de représentations imperceptibles, les stoïciens ajoutaient toute une série d'arguments sur tout ce que nous devons aux perceptions: sans perception, il ne pourrait y avoir selon eux ni arts ou techniques, ni mémoire, ni vertu, ni même d'actions, puisque nous ne donnerions notre assentiment à rien et resterions inactifs (Cicéron, $A c$. Pr. II, 19-25). Cette série d'arguments sur la dépendance de l'art et de la vertu (qui est une forme de techne) à l'égard de la perception est cohérente à l'intérieur du système stoïcien, car elle suppose la définition stoïcienne de l'art comme «système de perceptions exercées ensemble relatives à l'un des buts utiles de la vie $\gg^{83}$. Mais elle était pour cette raison purement interne au système stoïcien, car elle reposait entièrement sur cette compréhension de l'art comme une forme de compréhension. Il suffisait aux académiciens de leur opposer les arts qui recouraient à la conjecture et ceux qui suivent le vraisemblable (Cicéron, $A c$. Pr. II, 107). Bien que Cicéron, dans ces arguments, reste allusif, il est facile de voir qu'une conception de la rhétorique comme une simple routine, telle qu'elle est développée dans le Gorgias, pouvait servir de base à l'argumentation des académiciens sur ce point. Quant à l'argument de l'action qui dépend de la compréhension, c'était un des points d'achoppement essentiels de la controverse, et il est frappant que la position de chacun des antagonistes soit présentée de façon inexacte par l'autre, d'une manière qui en vient à masquer les proximités, et ressemble à un dialogue de sourds. En $A c$. $P r$. II, 37-39, Cicéron présente l'argument que les stoïciens opposent aux académiciens sous une forme déconcertante : la compréhension ne peut se faire sans l'assentiment, qui est en notre pouvoir ; mais l'action non plus ne peut se faire sans assentiment préalable ; si on supprime l'assentiment, il n'y a donc ni compréhension ni action. Cicéron présente donc les choses comme si compréhension, assentiment et action étaient indissociables : on ne peut agir sans assentiment (ce qui est effectivement la doctrine stoïcienne), on ne peut avoir de perception sans assentiment (ce qui est aussi la doctrine stoïcienne), et donc on ne peut agir sans perception. Cette conclusion est manifestement incorrecte, car on peut très bien donner son assenti-

44 ; 196-197). L'intérêt de Zénon pour la résolution des sophismes est attesté (Plutarque, Stoic. Repugn. 8, 1034 E-F ; Diogène Laërce, VII, 16;25), mais rien ne prouve qu'il ait discuté de ces sophismes-là.

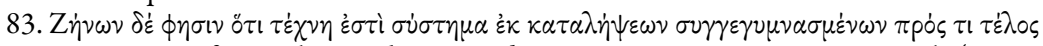

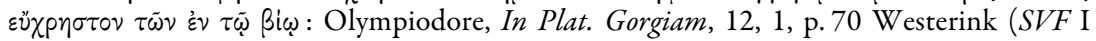
731). Voir Gourinat 2011. 
ment et agir en conséquence sans que cet assentiment soit perceptif. Certes, cela supprime la possibilité de l'action vertueuse, car celle-ci repose sur la science et donc sur la compréhension, qui est le préalable de la science. Mais cela n'entraîne pas pour autant l'inaction. Ce qui est paradoxal, c'est que stoïciens et académiciens ont polémiqué sur ce point alors qu'ils semblent en réalité d'accord pour dissocier l'action de la perception.

Carnéade objectait en effet à l'argument qui faisait reposer l'action sur la perception que, pour agir, le sage n'a besoin que de représentations crédibles $^{84}$ et $\ll$ non empêchées ${ }^{85}$. C'est ce qui avait amené Carnéade à distinguer entre un critère d'action, qui est la représentation crédible, et un critère de vérité, qui est la représentation perceptive des stoïciens. Carnéade admettait l'existence de la représentation crédible comme critère d'action mais rejetait l'existence de la représentation perceptive comme critère de la vérité. Cela lui permettait d'esquiver l'objection stoïcienne sur l'impossibilité d'agir dans le cadre du scepticisme. De cette position, nous avons deux témoignages, celui de Cicéron et celui de Sextus, $M$. VII, 166-189. D’après Sextus, Carnéade distinguait trois critères, un critère trivial, la représentation crédible $(\dot{\eta} \pi \imath \theta \alpha \nu \grave{\eta} \phi \alpha \nu \tau \alpha \sigma i \alpha)$, un critère pour les choses importantes, la représentation crédible non empêchée ( $\dot{\eta} \dot{\alpha} \pi \varepsilon p i \sigma \pi \alpha \sigma \tau \circ \varsigma \phi \alpha \nu \tau \alpha \sigma i \alpha)$, et un critère pour ce qui touche au bonheur, la représentation entièrement parcourue ( $\dot{\eta} \delta เ \varepsilon \xi \omega \delta \varepsilon \nu \mu \varepsilon \dot{\varepsilon} \eta \eta \phi \alpha \nu \tau \sigma \sigma \alpha)$. La représentation crédible s'oppose à la représentation incroyable, celle qu'Oreste a d'Électre dans sa folie. Certaines représentations crédibles sont obscures (par exemple, celles des objets éloignés) et ne constituent pas un critère. Seules les représentations crédibles vives sont des critères, mais ce critère se révèle parfois faux, de sorte qu'on ne peut pas lui donner cet assentiment inébranlable qui est la marque de la perception chez les stoïciens. Le point de départ de l'analyse de Carnéade en ce qui concerne la représentation non empêchée (quae non impediatur) est qu'une représentation n'est jamais isolée, elle est donnée dans un contexte ( $\tau \dot{\alpha} \dot{\varepsilon} \kappa \tau \dot{s} \varsigma)$ qui peut provoquer une suspicion : l'air, la lumière, le jour, le ciel, la terre, les amis, « et tout le reste » peuvent nous empêcher d'accepter comme critère une représentation crédible. C'est dans ce cadre que Carnéade donne l'exemple de Ménélas rencontrant Hélène à la cour de Protée. Il souligne l'importance de la convergence des représentations (бuv-

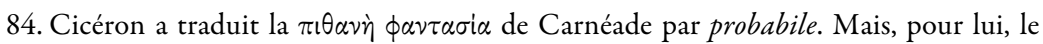
terme signifie seulement : «ce qui est susceptible d'être approuvé », sans qu'il y ait la dimension statistique que nous mettons dans la probabilité. «Représentation crédible » serait une traduction plus fidèle. Sur le problème de la traduction de Cicéron, voir Schofield 1999 , p. 350.

85. Cicéron, Ac. Pr. II, 33 ; 99-107. 
$\delta p o \mu \eta ̀ ~ \phi \alpha \nu \tau \alpha \sigma i \tilde{\omega} \nu)$. S'il y a des empêchements, la représentation n'est pas un critère.

Carnéade, selon Cicéron, Ac. Pr. II, 99, pensait qu'«aucune représentation n'avait qualité à entraîner une perception (perceptio) », mais que «beaucoup en revanche suscitaient une approbation (probatio) ». Il règle

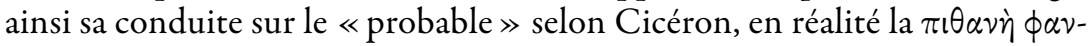
$\tau \alpha \sigma i \alpha$ ou représentation crédible, c'est-à-dire celle qui est susceptible d'être crue ou de recevoir notre adhésion. Selon Cicéron, Carnéade lui-même ou d'autres académiciens ont tiré argument de la conduite du sage stoïcien pour justifier de régler ses actions sur le probable :

Ainsi, tout ce qui se présentera sous un aspect probable, si rien ne s'offre de contraire à cette probabilité, le sage en fera usage, et toute sa conduite sera gouvernée de cette manière. Même le sage que vous mettez en scène suit souvent beaucoup de choses probables (multa probabilia), non comprises (non comprehensa), ni perçues (neque percepta), auxquelles il n'a pas donné son assentiment (neque adsensa), mais qui sont semblables au vrai (similia veri), car s'il ne les approuvait pas, toute vie lui serait ôtée. (100) Eh quoi ? en montant sur un navire, le sage comprend-il par son esprit et perçoit-il (comprehensum animo atque perceptum) que la traversée se fera selon son avis ? Comment le pourrait-il ? Mais s'il partait d'ici pour Pouzzoles, qui n'est qu'à trente stades, avec un équipage fiable, un bon pilote, par une mer calme, il lui paraîtrait probable (probabile videatur) qu'il arriverait là-bas sans dommage. Il prendra donc conseil de représentations de ce genre pour agir ou ne pas agir. (Cicéron, Ac. Pr. II, 99-100.)

De fait, il semble bien que les stoïciens aient recommandé de régler l'action sur le probable, faute de pouvoir la régler sur le certain. Le terme

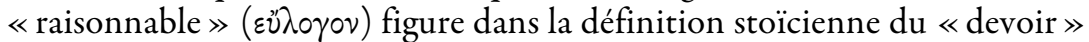
( $\kappa \alpha \theta \tilde{\eta} \kappa \circ \nu)$ dès Zénon ${ }^{86}$, et semble avoir été repris comme critère d'action par Arcésilas ${ }^{87}$. Un passage de Philodème citant le stoïcien Dionysios ${ }^{88}$ et un passage de Sénèque confirment l'importance du raisonnable en reprenant l'exemple de la navigation:

Il dit d'abord [...] qu'il nous suffira, concernant ces choses et celles qui dérivent de l'expérience, d'être convaincus $(\pi \varepsilon \pi \varepsilon \tilde{\sigma} \sigma \alpha \iota)$ selon le raisonnable $(\kappa \alpha \tau[\grave{\alpha} \tau \dot{\eta} \nu] \varepsilon \dot{\nu} \lambda \circ \gamma i \alpha \nu)$, tout comme nous le sommes quand nous prenons la

86. Diogène Laërce, VII, 107-108.

87. Sextus, $M$. VII, 158. Les interprètes sont divisés quant à savoir si Arcésilas reprenait le terme de manière purement dialectique à Zénon ou s'il l'avait repris à son propre compte : voir Ioppolo 1986, p. 121-131 ; Schofield, 1999, p. 332-334 ; Ioppolo 2009, p. 109-130.

88. Il s'agit de Dionysios de Cyrène, stoïcien disciple de Diogène de Séleucie et d'Antipater (voir Dorandi 1994, $D P h A$ D 180). 
mer en plein été, que nous arriverons sains et saufs. (Philodème, De signis, VII, 32-35 = 11, 4-6.)

Nous n'attendons jamais une compréhension absolument certaine des choses (certissimam rerum comprehensionem), parce que la recherche de la vérité est une chose ardue, et nous empruntons le chemin que guide ce qui est semblable au vrai (veri similitudo). Telle est notre voie dans tout devoir que nous entreprenons: c'est ainsi que nous semons, que nous naviguons, que nous faisons la guerre, que nous nous marions, que nous élevons nos enfants. Bien que, dans toutes ces entreprises, le résultat soit incertain, nous nous décidons pour des actes dans lesquels nous croyons pouvoir espérer. Car qui promettra au semeur une moisson, au marin un port, au militaire une victoire, au mari une épouse chaste, au père des enfants respectueux ? Nous suivons le chemin sur lequel nous conduit la raison, non la vérité. (Sénèque, Ben., IV, 33, 2-3.)

L'une des différences, c'est que la «probabilité » stoïcienne est une probabilité dans l'estimation des événements futurs et du résultat de nos actions, tandis que la représentation «crédible » de Carnéade vise même la réalité actuelle. Cicéron traduit par le même terme, probabilis, le « raisonnable » ou «probable » stoïcien ( $\varepsilon \dot{\lambda} \lambda \circ \gamma \circ v)$, qui correspond à une évaluation raisonnable et contient une part d'évaluation objective ${ }^{89}$ et la repré-

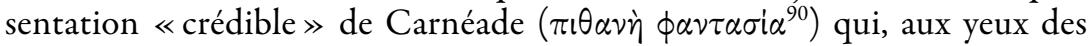
stoïciens, n'est crédible qu'en ce sens qu'elle induit à l'assentiment ${ }^{91}$. Dans l'argument carnéadien du Lucullus, II, 99-100, Cicéron fait comme si les stoïciens avaient reconnu comme critère d'action le probable/crédible de Carnéade, alors que c'est le probable/raisonnable qui leur sert de critère d'action. Il est vrai que la position que Philodème prête à Dionysios, selon qui nous devons être « convaincus $(\pi \varepsilon \pi \varepsilon \tilde{\tau} \sigma \theta \alpha$ l) selon le raisonnable », semble autoriser cette confusion jusqu'à un certain point, mais, même là, les notions restent distinctes. D'autre part, les stö̈ciens admettent un critère de la vérité différent du critère d'action, la représentation perceptive, alors que Carnéade s'en tient au probable comme critère d'action et de «con-

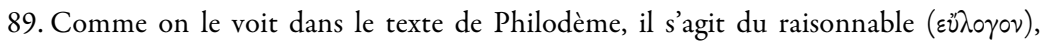
c'est-à-dire pour les stö̈ciens de ce qui a plus de probabilités statistiques d'être vrai que d'être faux, comme : «Je serai encore en vie demain » (Diogène Laërce, VII, 76). « La proposition raisonnable est différente de la représentation perceptive », dit Diogène Laërce, VII, 177, commentant une anecdote mettant en scène Sphaïros du Bosphore qui se laisse abuser par des grenades en cire et se défend en disant qu'il a cru qu'il était « raisonnable » que ce soient des grenades en cire.

90. Sextus, $M$. VII, 166.

91. «Une proposition crédible $(\pi \imath \theta \alpha \nu o ̀ \nu ~ \grave{\alpha} \xi \omega \mu \alpha)$ est une proposition qui conduit à l'assentiment, comme : "Si un être a mis bas quelque chose, il en est la mère", mais c'est faux, car l'oiseau femelle n'est pas la mère de l'œuf. » (Diogène Laërce, VII, 75.) 
naissance » incertaine. On a donc l'impression dans le Lucullus d'assister à un dialogue de sourds entre stoïciens et académiciens qui n'est dû qu'à un choix de traduction qui supprime la différence entre deux termes qui, aux yeux des stoïciens, ont des significations très différentes.

\section{Les principes de la polémique \\ Le principe d'Épicure}

L'un des arguments évoqués par Cicéron est un principe dont il avoue qu'il est emprunté à Épicure :

Si unus sensus semel in vita mentitus sit, nulli umquam esse credendum, «si un sens nous a menti une fois dans la vie, il ne faut plus jamais en croire aucun. » (Ac. Pr. II, 79-80.)

Si ullum sensus visum falsum est, nibil percipi potest, « si une seule représentation sensible est fausse, rien ne peut être perçu. » (Ac. Pr. II, 101.)

Ce principe sera repris par Descartes :

Prudentiae est numquam illis plane confidere qui nos semel deceperunt (Med. I, AT VII 18).

Il est de la prudence de ne se fier jamais entièrement à ceux qui nous ont une fois trompés (Méd. I, AT IX 14).

C'est ce principe qui justifie la transformation du rejet de certaines représentations fausses en doute et en suspicion généralisée à l'égard de toutes nos représentations, puisque les stoïciens admettent qu'il existe des représentations sensibles fausses. Cicéron imagine une réponse stoïcienne extrêmement simple: Non concedo Epicuro, « je ne le concède pas à Épicure. » (Ac. Pr. II, 101.)

En un sens, il n'y a rien à dire de plus. Les académiciens empruntent, pour critiquer les stoïciens, un argument épicurien. Ce n'est pas une réfutation ad hominem, puisque ce principe n'est pas admis par les stö̈ciens. Selon les règles de la dialectique, ce n'est même pas une réfutation du tout, puisque précisément, dialectiquement, il n'y a rien à tirer d'un principe que l'interlocuteur n'admet pas. Aussi bien Cicéron se contente-t-il de renvoyer épicuriens et stoïciens dos à dos, ce qui est une attitude académicienne ${ }^{92}$. Il ne prouve pas que les stoïciens ont tort. Il montre que la position stoïcienne (il y a des représentations fausses et des représentations perceptives) et la position épicurienne (toutes nos représentations sont vraies) sont incompatibles. Ce désaccord entre les écoles produit l'abstention académicienne. Mais cela ne réfute pas véritablement l'existence de la perception. Or la polémique sur la représentation perceptive repose effectivement en grande 
partie sur l'idée que, puisque nous nous laissons parfois abuser par des représentations fausses ou des hallucinations, il n'est pas possible de se fier à nos représentations perceptives. Elle repose donc bien sur un argument non concédé par les stoïciens, de sorte que la position stoïcienne n'est pas réfutée.

\section{Le continuisme perceptif des stoüciens comme clé de la position stoücienne}

L'un des points de divergence entre stoïciens et académiciens apparaît dans le traitement de la question des hallucinations. Pour les stö̈ciens, l'argument de l'hallucination n'est pas probant, puisque, après le rêve ou après la crise de délire, celui qui était sujet à l'hallucination cesse de lui donner son assentiment. Les académiciens n'admettent pas que ce soit un argument recevable : «Ce qui est en question, c'est à quoi ressemblent les choses quand elles apparaissent. » (Cicéron, Ac. Pr. II, 88.)

La question n'est pas celle de la recordatio, du souvenir que nous avons de nos hallucinations et de nos représentations lorsque nous recouvrons nos esprits après la crise, c'est celle de la qualité de la représentation (qualis visio) au moment où elle se produit (II, 90).

Il y a en fait une différence de perspective entre le point de vue stoïcien et le point de vue académicien. Les académiciens visent au fond une espèce d'image passive et statique, que l'on examine sous tous ses aspects. C'est ce qui apparaît assez clairement dans le critère de la représentation «parcourue » de Carnéade. Comme si la représentation était une image qu'il suffisait de regarder avec suffisamment d'attention pour la parcourir entièrement. Les stö̈ciens refusent clairement l'idée que la représentation soit ainsi une image statique. La représentation suppose un mouvement continu, et donc un continuisme perceptif.

C'est l'un des aspects de la polémique de Chrysippe contre Cléanthe. Zénon avait défini la représentation comme une impression dans l'âme (Sextus, M. VII, 236). Cléanthe, tributaire de l'emprunt que Zénon avait fait à la métaphore du bloc de cire dans le Théétète, $191 \mathrm{~d}-\mathrm{e}^{93}$, et pensant qu'il fallait prendre le terme au pied de la lettre, interpréta la représentation comme une «forme en creux et en pleins, comme l'impression du sceau dans la cire » $(M$. VII, 228). Chrysippe remarqua qu'il serait impossible que l'âme se représente plusieurs choses à la fois ou conserve une représentation en mémoire: «le dernier mouvement obscurcirait toujours la représentation précédente, de même que l'impression d'un second sceau oblitère toujours le premier » $(M$. VII, 229). Pour lui, la représentation n'est donc pas une empreinte en creux et en pleins, mais une « modification » ou une 
«altération» de l'âme comparable à la façon dont l'air, «lorsque différentes personnes parlent en même temps, est frappé au même moment de coups différents et innombrables, c'est-à-dire de modifications $\gg(M$. VII, 230). L'interprétation de Cléanthe, tributaire d'une interprétation littérale de la métaphore de l'impression, impliquait une conception discontinuiste de la vision, similaire à celle des épicuriens, pour qui la vision se produit parce que de petits effluves se détachant des corps, qui sont comme des images ou simulacres, viennent frapper nos yeux. Cela suppose que nous ne voyons des corps en mouvement que parce que les effluves qui s'en détachent frappent successivement nos yeux (Lucrèce, IV, 794-801), exactement comme dans la vision cinématographique où nos yeux ne reçoivent qu'une succession d'images fixes mais perçoivent des mouvements par persistance rétinienne. L'explication de la représentation par une succession d'empreintes renvoie au même mécanisme. Nous n'aurions pas de représentation continue à travers le temps.

La conception de Chrysippe suppose au contraire une continuité de la représentation, en accord avec le continuisme général de la physique stoïcienne. Il n'y a pas plus de discontinuité entre les représentations qu'il n'y a de vide dans la nature. Le test de Carnéade est ainsi quasi synchronique, alors que celui de Chrysippe est diachronique. Il faut du temps pour percevoir et la perception ne se fait pas en un instant :

C'est pourquoi nous voulons souvent que la lumière soit modifiée, ainsi que la situation des objets que nous regardons; c'est pourquoi nous diminuons ou nous augmentons les intervalles, et nous faisons beaucoup de choses jusqu'à ce que l'aspect de la représentation nous donne lui-même confiance dans le jugement qui s'y rapporte. (Cicéron, $A c$. Pr. II, 19.)

La conception stoïcienne de la perception vise en définitive un acte extrêmement complexe, qui ne suppose pas une simple représentation instantanée, mais un processus inscrit dans le temps, qui implique des affects psychiques continus. Ce n'est pas une image que nous percevons, ni une succession d'images, c'est une modification affective de l'âme, qui est un mouvement ${ }^{94}$, une impression, et non une empreinte figée. La gravure figée du Théétète est remplacée par une image en mouvement. Nous ne comprenons ou ne percevons ainsi une chose que lorsque nous aurons donné notre assentiment à une représentation qui aura acquis la force de l'évidence. Dans la conception stoïcienne, la continuité du processus de représentation et l'adjonction d'un assentiment sont indissociables de la notion de perception. 
Il apparaît donc clairement que c'est en ayant encore cette conception stoïcienne à l'esprit que Descartes écrira :

Atque ita quod putabam me videre oculis, solâ judicandi facultate, quae in mente meâ est, comprehendo. (Med. II, AT VII 32.)

Et ainsi je comprends par la seule puissance de juger, qui réside en mon esprit, ce que je croyais voir de mes yeux. (Méd. II, AT IX 25.)

Pour lui comme pour les stoïciens, comprendre ou percevoir suppose un acte d'assentiment de l'esprit. Descartes en changera le sens, mais sans la conception stö̈cienne, il n'aurait sans doute jamais conçu les choses ainsi. Notre notion moderne de la perception, telle qu'elle s'est construite depuis Descartes, est donc tributaire de l'analyse stoïcienne et de la contribution académicienne au débat, sauf sur ce point qu'elle fait souvent de l'impression une simple image statique, alors qu'il s'agit dans le stoïcisme d'une image en mouvement.

Mais qui a raison? Y a-t-il ou non des perceptions? Y a-t-il des représentations perceptives, discernables des représentations fausses et des hallucinations? La balance, même chez les adversaires des stoïciens, a souvent penché en leur faveur. Voici en effet les lignes que Cicéron écrivit à Atticus, où les arguments d'Antiochus reprennent ceux des stö̈ciens :

Je ne suis pas parvenu à faire en sorte que ma cause paraisse supérieure. Car les arguments d'Antiochus sont fortement crédibles : exprimés par moi scrupuleusement, ils ont la pénétration d'Antiochus et le brillant de mon style, si tant est que j'en aie. (Cicéron, Att. XIII, 19, 5.) 


\begin{tabular}{|c|c|c|}
\hline \multicolumn{3}{|c|}{$\begin{array}{l}\text { ANNEXE } \\
\text { Tableau }\end{array}$} \\
\hline & $\begin{array}{l}\text { Antiochus/stoïciens [ou } \\
\text { épicuriens] }\end{array}$ & académiciens sceptiques \\
\hline \multicolumn{3}{|c|}{ Erreurs des sens } \\
\hline $\begin{array}{l}\text { a) cou du pigeon } \\
\text { [exemple épicurien] }\end{array}$ & $\begin{array}{l}\text { Ac. Pr. II, } 19 \\
\text { il faut varier les conditions } \\
\text { (éclairage, distance) }\end{array}$ & $\begin{array}{l}\text { Ac. Pr. II, } 79 \\
\text { plusieurs couleurs vues, une } \\
\text { seule réelle }\end{array}$ \\
\hline $\begin{array}{l}\text { b) rame tordue dans l'eau } \\
\text { [exemple épicurien] }\end{array}$ & $\begin{array}{l}\text { Ac. Pr. II, } 19 \\
\text { M. VII, } 244\end{array}$ & $\begin{array}{l}\text { Ac. Pr. II, } 79 \\
\text { Ac. Pr. II, } 82 \\
\text { [Aug. Trin. XV, 12, 21] }\end{array}$ \\
\hline $\begin{array}{l}\text { c) bâtiments immobiles } \\
\text { paraissant bouger depuis un } \\
\text { bateau [exemple épicurien] }\end{array}$ & & Ac. Pr. II, 81-82 \\
\hline $\begin{array}{l}\text { d) taille du soleil [exemple } \\
\text { épicurien] }\end{array}$ & $\begin{array}{l}\text { repris par les stoïciens selon } \\
\text { Calcidius, in Tim., } 237\end{array}$ & Ac. Pr. II, 82 \\
\hline \multicolumn{3}{|c|}{ Représentations déformées (folie) } \\
\hline $\begin{array}{c}\text { Oreste prend Électre pour } \\
\text { une Érynie }\end{array}$ & $M$. VII, 244-245 & \\
\hline $\begin{array}{l}\text { Hercule prend ses enfants } \\
\text { pour ceux d'Eurysthée }\end{array}$ & $M$. VIII, 67 & $\begin{array}{l}\text { Ac. Pr. II, } 88 \\
M . \text { VII, } 405 \\
\end{array}$ \\
\hline \multicolumn{3}{|c|}{ Visions vides } \\
\hline $\begin{array}{l}\text { a) dormeurs } \\
\text { (rêve) }\end{array}$ & $\begin{array}{l}\text { Ac. Pr. II, 51-52 } \\
M \text {. VII, 244-245; } 247 \\
\text { manque de clarté + réveil }\end{array}$ & $\begin{array}{l}\text { Ac. Pr. II, } 48,88 \\
M . \text { VII, } 403-404 \\
\text { [Aug. Trin. XV, 12, 21] }\end{array}$ \\
\hline $\begin{array}{l}\text { b) ivrognes } \\
\text { (délire éthylique) }\end{array}$ & $\begin{array}{l}\text { Ac. Pr. II, 51-52 } \\
\text { assentiment faible }\end{array}$ & Ac. Pr. II, 88 \\
\hline $\begin{array}{l}\text { c) fous } \\
\text { (crises de folie) }\end{array}$ & $\begin{array}{l}\text { Ac. Pr. II, 52-53 } \\
\text { M. VIII, } 67\end{array}$ & $\begin{array}{l}\text { Ac. Pr. II, 88-89 } \\
\text { [Aug. Trin. XV, 12, 21] }\end{array}$ \\
\hline \multicolumn{3}{|c|}{ Objets indiscernables } \\
\hline a) jumeaux & $\begin{array}{l}\text { Ac.Pr. II, } 56 \\
\text { mère qui reconnaît ses } \\
\text { jumeaux }\end{array}$ & Ac. Pr. II, 85 \\
\hline b) œufs & $\begin{array}{l}\text { Ac. Pr. II, } 57 \\
\text { éleveurs de poules à Délos }\end{array}$ & $\begin{array}{l}\text { Ac. Pr. II, } 86 \\
\text { M. VIII } 409\end{array}$ \\
\hline c) objets fabriqués en série & & Ac. Pr. II, $85-86$ \\
\hline \multicolumn{3}{|c|}{ Propositions indiscernables } \\
\hline a) sorites & Ac. Pr. II, 49-50 & Ac. Pr. II, 93-94 \\
\hline b) argument du menteur & & Ac. Pr. II, 98 \\
\hline
\end{tabular}




\section{BIBLIOGRAPHIE}

BEYSSADE, J.-M. 2001 : Descartes au fil de l'ordre, Paris, 2001 (Épiméthée).

BRENNAN, T. 2005 : The Stoic Life: Emotions, Duties, and Fate, Oxford, 2005.

BRÉHIER, É. 1962 : Les Stoïciens, textes traduits par É. Bréhier, édités sous la direction de P.M. Schuhl, Paris, 1962 (Bibliothèque de la Pléiade).

Brittain, C. 2006 : Cicero, On Academic Scepticism, translated with introduction and notes, Indianapolis/Cambridge, 2006.

- 2012: «Antiochus' epistemology » dans D. Sedley (éd.), The Philosophy of Antiochus, Cambridge, 2012, p. 104-130.

Couissin, P. 1929: «Le Stoïcisme de la Nouvelle Académie », Revue d'histoire de la philosophie, 3 (1929), p.241-276. [Traduction anglaise par J.Barnes et M. Burnyeat, « The Stoicism of the New Academy », dans M. Burnyeat (éd.), The Skeptical Tradition, Berkeley-Los Angeles-Londres, 1983, p. 31-63.]

Dorandi, T. 1994 : «Dionysios de Cyrène », D 180, dans R. Goulet (dir.), Dictionnaire desphilosophes antiques, II, Paris, 1994, p. 865-866.

Duнот, J.-J. 1991: «Y a-t-il des catégories stö̈ciennes?», Revue internationale de philosophie, 45 (1991), p. 221-244.

FREDE, M. 1987 : « Stoics and Skeptics on clear and distinct impressions », dans Id., Essays in Ancient Philosophy, Oxford, 1987, p. 151-176 (= M. Burnyeat (éd.), The Skeptical Tradition, Berkeley-Los Angeles-Londres, 1983, p. 65-93).

— 1999: « Stoic epistemology », dans K. Algra, J. Barnes, J. Mansfeld \& M. Schofield (éd.), The Cambridge History of Hellenistic Philosophy, Cambridge, 1999, p. 295322.

Goulet-CAZÉ, M.-O. 2011 : «À propos de l'assentiment stoïcien », dans M.-O. GouletCazé (éd.), Études sur la théorie stoïcienne de l'action, Paris, 2011 (Textes et traditions, 22), p. 73-236.

Gourinat, J.-B. 1996 : Les stö̈ciens et l'âme, Paris, 1996 (Philosophies, 75).

- 2011: «Les définitions de l'epistêmê et de la technê dans l'ancien stoïcisme », dans J. Jouanna, M. Fartzoff, B. Bakhouche (éd.), L'homme et la science, actes du XVI Congrès international de l'Association Guillaume Budé, Paris, 2011, p. 243-256.

INwOOD, B. 1985 : Ethics and Human Action in Early Stoicism, Oxford, 1985.

IOPpOLO, A.M. 1986: Opinione e scienza. Il dibattito tra Stoici e Accademici nel III e nel II secolo a.C., Naples, 1986 (Elenchos, 12).

- 1990 : «Presentation and assent: a physical and cognitive problem in early Stoicism », Classical Quarterly, 40 (1990), p. 433-449.

- 1997 : « Fidelity to Zeno's theory », Diotima, 25 (1997), « Chypre et les origines du stoïcisme $\gg$, p. 62-73.

-2009: La testimonianza di Sesto Empirico sull'Accademia scettica, Naples, 2009 (Elenchos, 53).

Kany-Turpin, J. 2010 : Cicéron, Les Académiques / Academica, Traduction, notes et bibliographie par —, Introduction par P. Pellegrin, Paris, 2010 (GF Flammarion, 1460).

KeRFERD, G.B. 1978 : «The problem of synkatathesis and katalepsis in Stoic doctrine », dans J. Brunschwig (dir.), Les Stö̈ciens et leur logique: Actes du colloque de Chantilly, 18-22 sept. 1976, Paris, 1978 [2 éd. revue 2006] (Bibliothèque d'histoire de la philosophie), p. 251-272.

LONG, A. 2002: «Zeno's epistemology and Plato's Theaetetus», dans T. Scaltsas \& A. Mason (éd.), The Philosophy of Zeno, Larnaca, 2002, p. 115-131.

LONG, A. \& D. SEDLEY 1987 : The Hellenistic Philosophers, Cambridge, 1987 (2 vol.). 
MetTe, H.J. 1984: «Zwei Akademiker heute: Krantor von Soloi und Arkesilaos von Pitane », Lustrum, 26 (1984), p. 7-94.

Sandbach, F.H. 1971: «Phantasia Kataleptike», dans A. Long (éd.), Problems in Stoicism, Londres, 1971, p. 9-21.

SCHOfIELD, M. 1999 : « Academic epistemology », dans K. Algra, J. Barnes, J. Mansfeld \& M. Schofield (éd.), The Cambridge History of Hellenistic Philosophy, Cambridge, 1999, p. 323-351.

SEDLEY, D. 2005: «La définition de la phantasia katalêptikê par Zénon», dans J.B. Gourinat (éd.), Les stö̈ciens, études sous la direction de G. Romeyer Dherbey, Paris, 2005 (Bibliothèque d'histoire de la philosophie), p. 75-92.

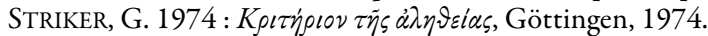

- 1990: «The problem of the criterion », dans S. Everson (éd.), Companions to Ancient Thought, 1, Epistemology, Cambridge, 1990, p. 143-160.

- 1997 : «Academics fighting Academics », dans B. Inwood \& J. Mansfeld (éd.), Assent and Argument. Studies in Cicero's Academic Books: Proceedings of the 7th Symposium Hellenisticum (Utrecht, August 21-25, 1995), Leyde-New YorkCologne, 1997 (Philosophia Antiqua, 76), p. 257-276.

SVF : Stoicorum Veterum Fragmenta, collegit I. ab Arnim, Leipzig, 1903-1905, 3 vol. (réimpression Stuttgart, 1978-1979).

Tieleman, T. 2003 : Chrysippus on Affections, Leyde-Boston, 2003 (Philosophia Antiqua, 94).

VOELKe, A.-J. 1973: L'Idée de volonté dans le stö̈cisme, Paris, 1973 (Bibliothèque de philosophie contemporaine). 